15

\title{
Фокусирующая капиллярная и пористая рентгеновская оптика
}

\author{
() В.В. Лидер \\ Институт кристаллографиии им. А.В. Шубникова ФНИЦ „Кристаллография и фотоника“ РАН, \\ Москва, Россия \\ e-mail: vallider@yandex.ru
}

Поступила в редакцию 29.05.2021 г.

В окончательной редакции 29.05.2021 г.

Принята к публикации 21.07.2021 г.

Рассмотрены принципы работы и возможности рентгеновской фокусирующей капиллярной и пористой оптики. Особое внимание уделяется поликапиллярной оптике, конфокальной технике рентгеновского флуоресцентного анализа, а также широкоугольной оптике „глаза лобстера“.

Ключевые слова: рентгеновские лучи, фокусировка, капилляр, пористая оптика, оптика „глаза лобстера“.

DOI: $10.21883 /$ OS.2021.11.51647.19-21

\section{1. Введение}

За последние три десятилетия появилось большое количество публикаций, посвященных неразрушающему изучению конденсированных сред с помощью рентгеновского микропучка [1].

Конденсорная оптика, с помощью которой осуществляется формирование рентгеновского микропучка, традиционно представляет собой зонную пластинку Френеля большого диаметра с десятками тысяч зон [2]. Зонная пластинка Френеля имеет ограниченную полосу пропускания, что может быть полезно в приложениях, где необходимо выделение определенной спектральной линии лабораторного источника рентгеновского излучения, но является недостатком в случае использования синхротронного излучения (СИ). Кроме того, зонные пластинки Френеля хрупки и отличаются низкой эффективностью.

В последнее время получили развитие альтернативные типы конденсора (концентратора) на основе полых стеклянных капилляров. В фокусирующих капиллярах используется эффект полного внешнего отражения (ПВО) рентгеновских лучей (РЛ) от внутренних стенок каналов, имеющих форму эллипсоида или параболоида вращения. Эти оптические элементы ахроматические и, следовательно, не функционируют в качестве монохроматоров, как в случае зонных пластинок Френеля. Такие капилляры обеспечивают равномерное освещение образца полым конусом РЛ и формирование фокусного пятна размером $\sim 1 \mu \mathrm{m}[3]$.

Капиляры имеют ряд преимуществ по сравнению с зонными пластинками Френеля, используемыми в качестве конденсора [4]: 1) они более доступны, 2) позволяют осуществлять с помощью монохроматора перестройку энергии без необходимости перемещения конденсора при изменении энергии РЛ, 3) их эффективность в 3-15 раз больше эффективности зонных пластинок при отсутствии нежелательных высоких дифракционных порядков, 4) являются более надежными, более долговечными, 5) стойки к тепловой нагрузке или механическим повреждениям, 6) не требуют наличия диафрагмы, устанавливаемой вблизи фокуса для формирования необходимой полосы пропускания и, таким образом, не ограничивают размер держателя образца и возможность управления им.

Изогнутые специальным образом капилляры позволяют создавать системы многократного отражения, способные управлять направлением распространения РЛ. Собрав вместе большое количество капилляров, изогнутых с разным радиусом кривизны, и расположив их таким образом, чтобы они направляли излучение в одну точку, можно создать эффективную рентгеновскую линзу.

Однако эллипсоидальным поверхностям присущи серьезные внеосевые аберрации. Поэтому капиллярная оптика не является изображающей. Но для конденсора расфокусировка внеосевого излучения не имеет значения, пока источник достаточно мал, а распределение освещения по углам одинаково по площади образца.

Ситуация меняется, когда необходимо создать эффективную рентгеновскую изображающую оптику, используемую, например, в рентгеновских орбитальных телескопах. В них для уменьшения аберраций обычно применяются составные зеркальные системы скользящего падения, известные как системы Вольтера I [5,6], в которых излучение последовательно отражается от двух асферических поверхностей. Для увеличения эффективной площади сбора телескопа используются несколько коаксиальных и конфокальных зеркал, плотно вложенных друг в друга. Однако увеличение плотности упаковки зеркал приводит к увеличению массы оптики, что затрудняет вывод аппаратуры на околоземную орбиту. Пористая оптика позволяет значительно облегчить вес телескопа с сохранением плотности отражающих поверхностей, а значит, и его эффективности [7].

Одно из направлений развития фокусирующей пористой оптики основано на использовании пластин 


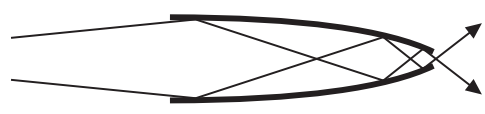

$b$

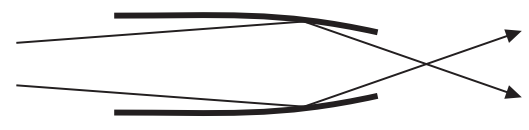

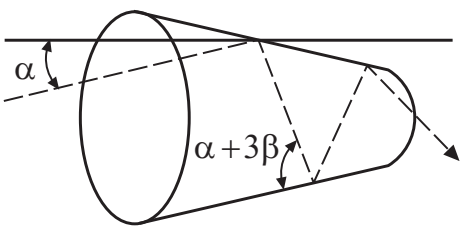

Рис. 1. Ход лучей в монокапилляре многократного (a), однократного (b) отражения и конусном капилляре [17] (c) (cм. текст).

кремния с ребрами жесткости. Пластины укладываются друг на друга, образуя каналы квадратного профиля субмиллиметрового размера. Такая конструкция может успешно заменить плотно упакованные зеркала в оптике Вольтера I, обеспечивая беспрецедентную легкость и жесткость конструкции.

Другое направление развития фокусирующей пористой оптики основано на использовании сферически изогнутой стеклянной микропористой пластины, и использование каналов квадратного профиля для фокусировки РЛ может стать эквивалентом так называемой оптики „глаза лобстера“ $[8]$.

\section{2. Фокусирующая капиллярная рентгеновская оптика}

В настоящее время рентгеновская капиллярная оптика является самостоятельным направлением в оптике [9]. Капиллярные рентгенооптические системы позволяют эффективно формировать рентгеновские пучки требуемой конфигурации в широком диапазоне энергий; они также просты по конструкции и технологичны в изготовлении. Очень важным свойством капиллярных оптических элементов является принципиальная возможность получения большей светосилы по сравнению с другими оптическими фокусирующими элементами рентгеновского диапазона, что позволяет использовать капиллярные системы в качестве эффективных концентраторов РЛ. В отличие от обычных методов фокусировки рентгеновского излучения капиллярная оптика дает возможность реально управлять пучками рентгеновских лучей [10-13].

Задача обрезания жесткой части электромагнитного излучения является проблемной в радиационной физике. Фильтрацию жесткой части спектра рентгеновского излучения можно осуществить с помощью капиллярных систем. Механизм фильтрации связан с тем фактом, что РЛ распространяются внутри каналов, отражаясь от их стенок, при условии малости скользящего угла $\theta$ $\left(\theta \leq \theta_{c}\right)$. Величина критического угла ПВО $\theta_{c}$ дается приближенной формулой:

$$
\theta_{c}(\mathrm{mrad}) \approx 2.34 \lambda(Z \rho / A)^{1 / 2},
$$

где $\rho\left(\mathrm{g} / \mathrm{cm}^{3}\right)$ - плотность материала, $Z$ и $A-$ соответственно его атомное число и вес, $\lambda(\AA)$ - длина волны РЛ.

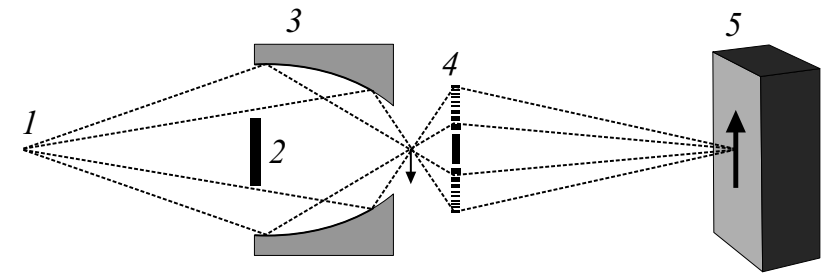

Рис. 2. Схема рентгеновского микроскопа полного поля зрения с эллипсоидальным капилляром в качестве конденсора: 1 - источник излучения, 2 - заглушка, 3 - капилляр, $4-$ объективная зонная пластинка Френеля, 5 - детектор [3].

Поскольку угол $\theta_{c}$ пропорционален длине волны РЛ, то, выбирая определенную геометрию оптической системы, можно добиться, чтобы для коротковолновой части спектра излучения нарушалось условие каналирования (распространения внутри канала), и пропускная способность системы была уменьшена. С другой стороны, в области больших длин волн пропускная способность системы также снижается из-за возрастающего поглощения излучения в этом диапазоне. Таким образом, каждая конкретная капиллярная система имеет естественную рабочую полосу пропускания.

Как показали проведенные исследования [14-16], качество внутренней поверхности стеклянных капилляров оказывается вполне пригодным для использования в рентгеновской оптике. Это означает, что такие капилляры могут служить эффективными волноводами для РЛ, т.е. транспортировать входящее в них излучение в достаточно широком спектре с потерями, существенными лишь при весьма большом числе отражений, поскольку потери на одно отражение можно свести к минимуму при соответствующем выборе материала капилляра и качестве его внутренней поверхности. Для типичных углов скольжения $\theta \sim \theta_{c} / 2$ высота шероховатостей, как правило, не должна превышать 10-20 А [9].

Очевидно, что рентгеновская капиллярная оптика может быть использована во всех областях, где необходимо увеличить плотность потока РЛ и где необходим контроль за их распространением.

\section{1. Монокапиллярная рентгеновская оптика}

Рисунок $1, a, b$ показывает различие между двумя типами монокапиллярной оптики: с многократным и однократным отражением. В первом случае РЛ после 


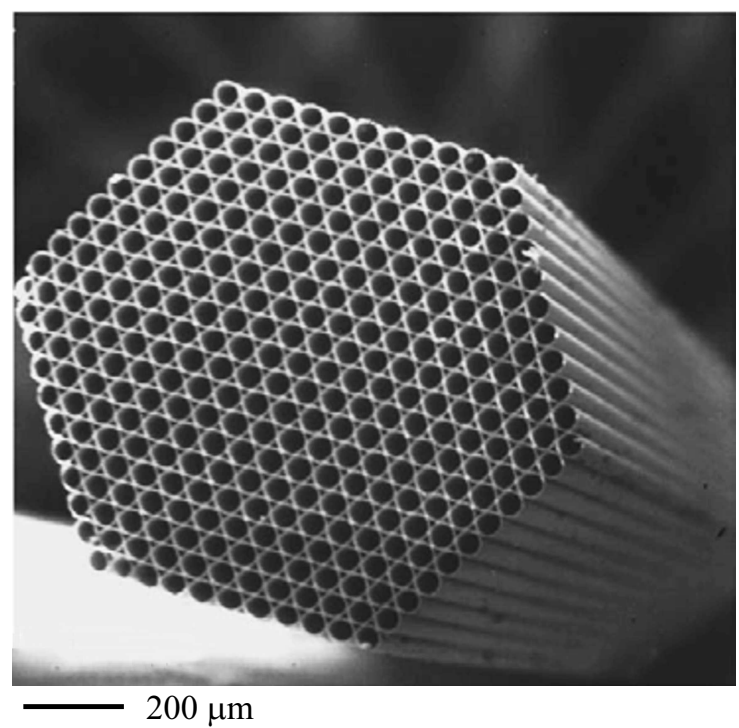

Рис. 3. Микрофотография сечения поликапиллярной линзы, полученного с помощью сканирующего электронного микроскопа. Диаметр каналов $\sim 50 \mu \mathrm{m}[55]$.

нескольких последовательных отражений формируют фокусное пятно, размер которого определяется размером выходного отверстия и свойствами источника излучения. Пропускная способность капилляра зависит от числа отражений, качества оптики и т. д., и обычно колеблется от 10 до 80\%. Недостаток - фокус находится в непосредственной близости от капиллярного выхода: обычно для получения малого размера фокуса образец должен быть помещен на расстоянии 10-100 $\mu \mathrm{m}$ для микронного размера фокусного пятна.

Для того чтобы иметь возможность получить расстояние фокуса от выходного отверстия, равное миллиметрам или сантиметрам, может быть использован монокапилляр однократного отражения (рис. 1, b). Он показывает почти 100\%-ю пропускную способность, так как единственное отражение очень эффективно в условиях ПВО [18].

Отметим, что из-за значительной расходимости пучка на выходе капилляра размер фокусного пятна $d_{f}$ всегда будет больше, чем выходной размер капилляра $d_{\text {out }}$ изза отличного от нуля фокусного расстояния $f$, т.е. от расстояния от конца капилляра до образца [14,19]:

$$
d_{f} \approx d_{\text {out }}+2 f \theta_{c} \text {. }
$$

Монокапилляры на основе однократного отражения обычно имеют эллиптическую или параболическую форму [20]. Используя эллиптический капилляр, точечный источник может быть сфокусирован, если источник расположен в одном из фокусов эллипса. Фокальное пятно тогда появится во втором фокусе. А параболический капилляр может фокусировать параллельный пучок или превратить расходящийся из точечного источника пучок РЛ в параллельный пучок. Размер фокального пятна будет определяться не только точностью изготовления оптического элемента, но также и свойствами источника излучения [21]. В работе [22] пучок РЛ с энергией $30 \mathrm{keV}$ был сфокусирован посредством эллиптического капилляра однократного отражения в фокусное пятно размером $15 \mu \mathrm{m}$.

Одним из способов получения рентгеновских пучков с субмикронными размерами является применение конусного капилляра, сужающегося по закону эллипса или параболы [17,23-27]. Авторами работы [25] было получено пространственное разрешение в $50 \mathrm{~nm}$ при $E=5-8 \mathrm{keV}$. Это - самое высокое разрешение, полученное с помощью рентгеновской монокапиллярной оптики.

В конусном капилляре (рис. 1,c) РЛ испытывают ПВО от гладкой внутренней поверхности капилляра при углах падения $\alpha$, меньших критического $\theta_{c}$. Первое отражение происходит под углом $\alpha+\beta$, где $\beta-$ половинный угол раствора капиллярного конуса. Второе отражение происходит под углом $\alpha+3 \beta$, а $n$-ное - под углом $\alpha+(2 n-1) \beta$. При падении под углом, большим критического, РЛ не участвует в процессе фокусировки. Таким образом, количество эффективных отражений определяется неравенством: $n<\left(\theta_{c}-\alpha+\beta\right) / 2 \beta[17,19]$.

Монокапиллярная оптика находит применение в качестве концентраторов РЛ с различными источниками излучения: СИ [3,28-31], рентгеновской трубки [32,33], лазерно-плазменных источников [34,35].

Капилляры-концентраторы с однократным отражением поддерживают широкий спектр приложений, включая рентгеновскую флуоресценцию [36-40] и томографию [41], рентгеновскую микроскопию [3,42-44] (рис. 2), малоугловое рассеяние РЛ [45], дифракционные методы $[28,45-48]$.

\section{2. Поликапиллярная рентгеновская оптика}

Простейшим элементом капиллярной оптики является прямой цилиндрический капилляр, представляющий собой полую стеклянную трубку цилиндрической формы. При помощи такого капилляра может быть осуществлено изменение направления распространения излучения, поскольку РЛ можно удержать в полом слабо изогнутом капилляре. При изгибе капилляра необходимо учитывать тот факт, что не все лучи, входящие в канал, будут удовлетворять условию $\theta<\theta_{c}$. В предположении малости внутреннего радиуса капилляра $r_{0}$ по сравнению с радиусом изгиба капилляра $R\left(r_{0} / R \ll 1\right)$ и с учетом малости критического угла ПВО для РЛ условие эффективного захвата излучения в режиме транспортировки излучения капилляром записывается как

$$
\left(R / r_{0}\right)\left(\theta_{c} / 2\right)^{2} \geq 1
$$

Таким образом, максимальный угол поворота рентгеновского пучка $\Psi_{\max }$ капилляром длиной $L$ определяется выражением

$$
\Psi_{\max }=\left(L / r_{0}\right)\left(\theta_{c} / 2\right)^{2} \text {. }
$$




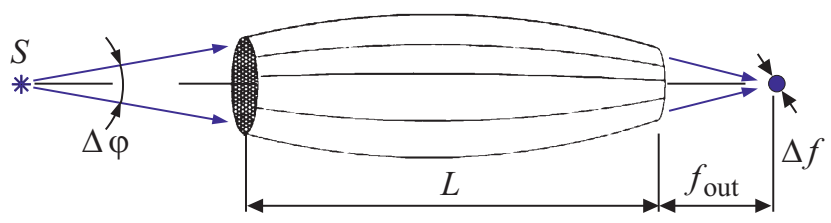

Рис. 4. Схема фокусировки рентгеновских лучей поликапиллярной линзой $(S-$ источник излучения, $\Delta \varphi-$ приемная апертура, $L-$ длина линзы, $f_{\text {out }}-$ фокусное расстояние, $\Delta f$ - размер фокусного пятна).

В литературе возможность использования капилляров в качестве элементов управления рентгеновским пучком описана и обоснована достаточно давно $[14,49]$. Было показано, что изогнутый капилляр принципиально способен поворачивать пучок жесткого рентгеновского излучения на углы до $10^{\circ}$ с эффективностью до 10\% [50].

Собрав вместе большое количество капилляров, изогнутых с разным радиусом кривизны, и расположив их таким образом, чтобы они направляли излучение в одну точку, можно создать рентгеновскую линзу. Такой системой и является поликапиллярная линза (оптика Кумахова), состоящая из множества изогнутых каналов [5156]. Для каждого канала, как и для зеркала, угол захвата излучения не превышает удвоенного критического угла, но каналов много, и реальный угол захвата линзы может достигать нескольких градусов, превосходя критический угол в десятки и сотни раз.

Условие $\theta<\theta_{c}$ требует использования каналов небольшого размера, обычно от 2 до $50 \mu \mathrm{m}$ (рис. 3). Изза механических ограничений производства тонкостенных трубок такого размера оптика изготавливается с использованием многократного процесса складывания и вытягивания стекловолоконной оптики [57].

Поликапиллярная система, спроектированная определенным образом, позволяет не только эффективно транспортировать излучение, но также и увеличивать плотность излучения, фокусируя его в пятно микронных размеров $\Delta f$. Следовательно, такая капиллярная система работает как фокусирующая линза. Предполагая идеальное перекрытие, размер фокусного пятна определяется размером пятна от каждого отдельного капилляра канала, который зависит от размера канала $r_{0}$, выходного фокусного расстояния $f_{\text {out }}$ (рис. 4 ) и локальной расходимости $\beta[55,58]$ :

$$
\Delta f \approx\left[r_{0}^{2}+\left(f_{\text {out }} \beta^{2}\right)\right]^{1 / 2} .
$$

Расходимость на выходе капилляра $\beta$ не равна нулю, но приблизительно определяется критическим углом и, следовательно, зависит от энергии рентгеновского излучения. Локальная расходимость $\beta$ обычно составляет $\sim 1.3 \theta_{c}$. Коэффициент $1.3-$ это экспериментально определенный параметр, который возникает из того факта, что большая часть пучка имеет расходимость меньше максимальной расходимости $2 \theta_{c}$, создаваемой отражением под критическим углом. Локальная расходимость пучка не зависит от размера источника, хотя большие источники не могут быть эффективно „захвачены“ оптикой. Критический угол $\theta_{c}$ при энергии излучения $E=20 \mathrm{keV}$ составляет $1.5 \mathrm{mrad}$. При $\beta=1.3 \theta_{c}$ оптика с $r_{0}=3.4 \mu \mathrm{m}$ и $f_{\text {fout }}=9 \mathrm{~mm}$ имеет прогнозируемый размер пятна $18 \mu \mathrm{m}$ [55].

Использование капиллярной линзы позволяет получить увеличение плотности излучения $G$, т. е. отношение плотности излучения в фокусном пятне линзы к плотности излучения, которая создается источником без линзы на том же расстоянии от источника, что и фокусное пятно [9]:

$$
G=(L \Delta \varphi / \Delta f)^{2} T,
$$

где $T$ - коэффициент пропускания поликапиллярной системы при условии, что пропускание системы не мало $(>10 \%), \Delta \phi-$ угловая апертура поликапилляра на входе, $L$ - длина линзы.

Сообщалось о $G=2500$ [59] и $G=2970$ [60] для стеклянного поликапилляра, что значительно больше коэффициента усиления конического монокапилляра

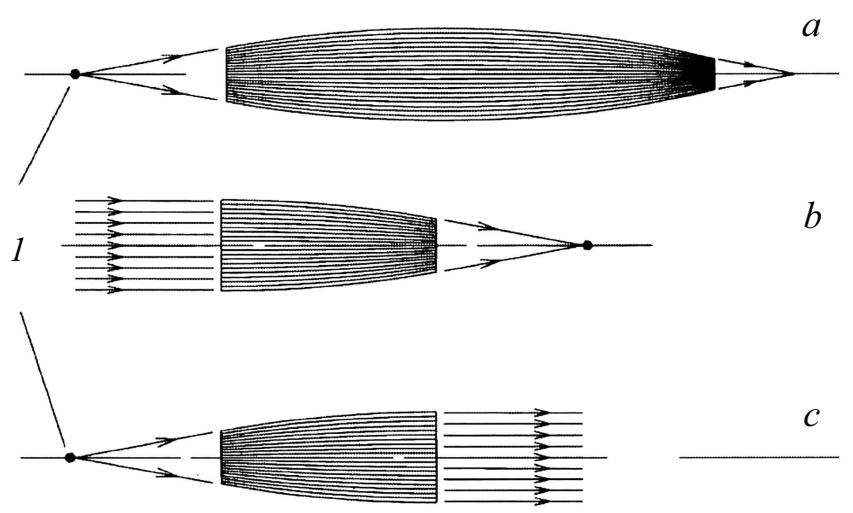

Рис. 5. Рентгеновская поликапиллярная оптика: фокусирующая линза „точечный источник-фокус“ $(a)$; фокусирующая полулинза „параллельный пучок-фокус“ $(b)$; полулинза „точечный источник-квазипараллельный пучок“ $(c)(1-$ точечный источник РЛ) [54].

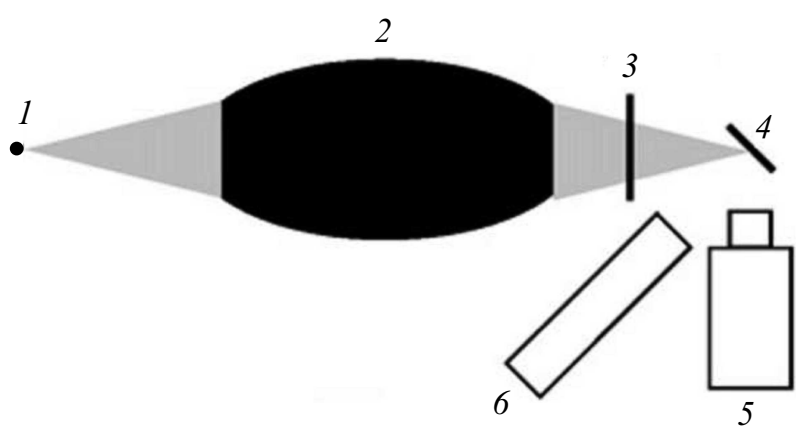

Рис. 6. Схема экспериментальной установки для рентгеновского флуоресцентного анализа $(1-$ анод микрофокусной рентгеновской трубки, 2 - поликапиллярная линза, 3 фильтр, 4 - образец, 5 - детектор, $6-$ оптический микроскоп) [60]. 
$(G=960$ при $E=6 \mathrm{keV})$, измеренного авторами работы [26].

Наряду с поликапиллярной линзой, позволяющей сфокусировать излучение от конечного источника (рентгеновской трубки), используется капиллярная полулинза, способная сфокусировать параллельный пучок (например, пучок СИ) или превратить его в квазипараллельный пучок с расходимостью в пределах удвоенного критического угла (рис. 5).

Было показано, что поликапиллярная фокусирующая линза совместно с рентгеновской микрофокусной трубкой может использоваться в медицине $[58,61]$, рентгеновской микроскопии $[62,63]$, для получения рентгеновского фазоконтрастного изображения [64], для микродифракции $[65,66]$, рентгеновского флуоресцентного анализа [60,67-69] (рис. 6).

Информация о двумерном (2D) распределении элементов может быть получена при растровом сканировании образца относительно сфокусированных в микропучок первичных РЛ. На сегодняшний день этот метод, часто называемый сканирующей рентгеновской флуоресцентной микроскопией (СРФМ), является наиболее популярным для исследований в области искусства и культурного наследия. Однако здесь могут возникнуть проблемы, связанные с большими размерами объектов исследования (например, произведений живописи), которые часто бывает затруднительно доставлять в лабораторию из музеев или хранилищ. Поэтому были разработаны мобильные установки с использованием поликапиллярной фокусирующей оптики для исследований на месте (in situ). Спрос на такие приборы стимулировал появление коммерческих инструментов [70]. Фирмой Bruker Nano GmbH (Берлин, Германия) были последовательно разработаны три варианта экспериментальных установок: ARTAX [71], Tornado M4 [72] и M6 Jetstream [70]. В них используются микрофокусные рентгеновские трубки и поликапиллярная фокусирующая оптика, обеспечивающая пространственное разрешение не хуже $100 \mu \mathrm{m}$. Система ARTAX была разработана, главным образом, для локального рентгеновского флуоресцентного анализа культурного наследия; также возможно ее использование для флуоресцентной визуализации [71], но область сканирования ограничена размером $5 \times 5 \mathrm{~cm}^{2}$. Таким образом, ARTAX не годится для изучения объектов больших размеров. В Tornado M4 максимальный размер исследуемого образца равен $20 \times 16 \mathrm{~cm}$, а для Bruker M6 Jetstream площадь сканирования достигла $80 \times 60 \mathrm{~cm}^{2}$; при этом осуществляется сканирование рентгеновским пучком неподвижного образца, что открывает возможности для исследования массивных объектов. Повидимому, в этом причина его повышенной востребованности [73-78].

Поликапиллярные полулинзы используются для эффективной фокусировки квазипараллельного пучка СИ с энергией в диапазоне $2-30 \mathrm{keV}$ до небольших пятен (диаметром 10-50 $\mu \mathrm{m}$ ) [79-85]. Поликапиллярные полулинзы также успешно используются в конфокальной рентгеновской оптике.

\section{3. Конфокальная рентгеновская оптика}

Одна из задач, стоящих перед исследователями, необходимость проведения анализа объектов с неоднородным распределением элементов не только по поверхности, но и по глубине, в частном случае объектов со слоистой структурой. Для решения этой задачи в начале 1990-х годов Гибсоном и Кумаховым $[53,86]$ был предложен метод конфокальной рентгеновской флуоресцентной спектроскопии (КРФС) [8790]. Сущностью метода является использование пространственной фильтрации для отсечения излучения от части образца вне фокуса (фоновых засветок). В КРФС помимо оптики, фокусирующей первичное излучение, используется дополнительный оптический элемент, установленный между образцом и детектором, обеспечивающий локальность отбора флуоресцентного излучения из объема образца. При этом регистрируется флуоресцентное излучение, поступающее только из объема, определяемого областью перекрытия оптических фокусов элементов рентгеновской оптики так называемого конфокального объема. Для этой цели обычно используется поликапиллярная линза или полулинза [91], позволяющая собрать флуоресцентное излучение и направить его на входное окно детектора (рис. 7, $a$ ).

Хотя использование СИ в соединении с конфокальным XRF показало его перспективность [92-94], ограниченная доступность инструментария стимулировала исследование в конфигурациях с лабораторными источниками $[89,95-102]$ (рис. 7,b). Настольные источники РЛ испускают намного меньше фотонов, чем источники СИ, что приводит к более низкой элементной чувствительности приблизительно на один-два порядка [103].

Однако разница в яркости источников излучения практически не влияет на разрешающую способность метода. Например, для оптической конфигурации, образованной двумя поликапиллярными линзами, для лабораторного инструмента [104] размер фокусного пятна был экспериментально оценен в $10 \mu \mathrm{m}$ при энергии $17.4 \mathrm{keV}\left(\right.$ Мо $\left.\kappa_{\alpha}\right)$, а разрешение по глубине варьировалось от 22.6 до $13.7 \mu \mathrm{m}$ при изменении энергии РЛ от 5.4 до $11.4 \mathrm{keV}$ соответственно (с увеличением энергии критический угол ПВО уменьшается и, таким образом, радиация может быть собрана из меньшего объема); при использовании СИ авторам работы [105] удалось получить конфокальный объем, равный $15 \times 15 \times 20 \mu \mathrm{m}^{3}$ при $E=17.2 \mathrm{keV}$. Заметим, что латеральное разрешение и разрешение по глубине определяются параметрами фокусирующей оптики, установленной перед образцом и перед детектором соответственно. 

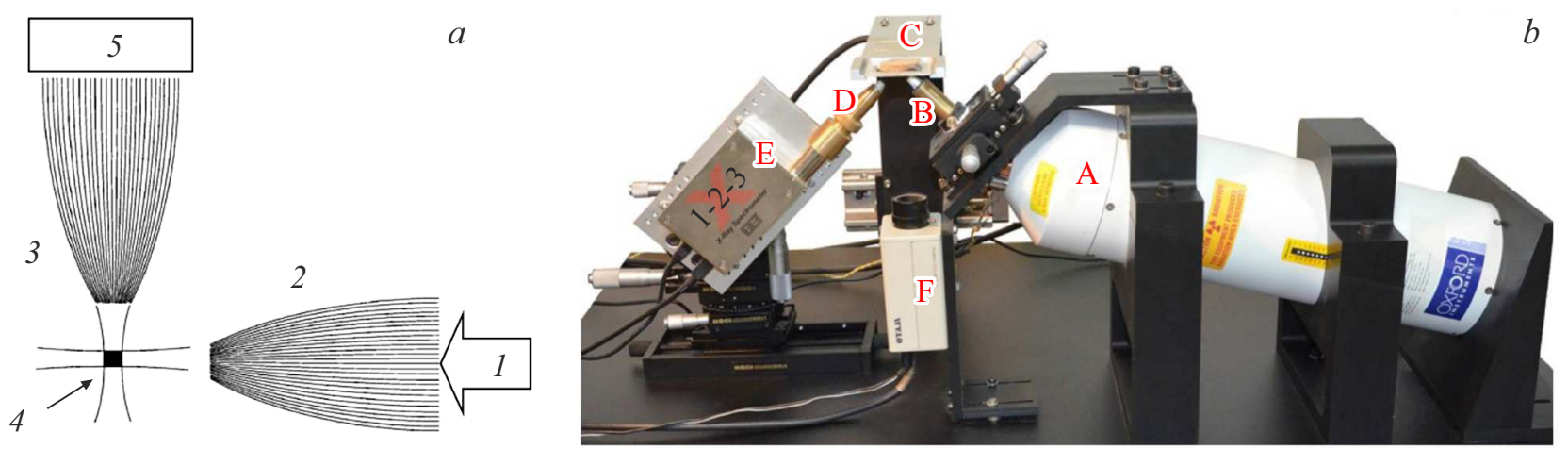

Рис. 7. (a) Схема рентгеновской конфокальной оптики при использовании СИ: 1 - квазипараллельный пучок СИ; 2 фокусирующая поликапиллярная полулинза; 3 - коллиматорная поликапиллярная полулинза, направляющая флуоресцентное излучение из конфокального объема 4 на детектор 5. (b) Лабораторный флуоресцентный спектрометр [101]: А - рентгеновская трубка, В - фокусирующая поликапиллярная линза, C - держатель образца, D - коллиматорная поликапиллярная полулинза, $\mathrm{E}$ - полупроводниковый детектор, F - CCD-камера.

Метод КРФС нашел применение в различных областях исследований. Разрешение по глубине играет принципиальную роль, когда последовательность слоев, состоящих из разных элементов, неизвестна, и если к тому же эта последовательность меняется вдоль поверхности образца. Поэтому метод использовался при изучении слоистых структур, таких как произведения живописи [106-116] и прикладного искусства [117119], многослойных полупроводниковых устройств [120]. Метод может применяться для изучения деталей механизмов [121], коррозии металлов [122,123], in operando преобразователей энергии $[124,125]$, границ раздела „жидкость/твердое тело“ [126]. Показана возможность использования метода КРФС в медицине [127-129], геологии [130-132], археологии [133-135], криминалистике $[136,137]$, изучении печатной продукции [138], исследовании окружающей среды [139], околоземного пространства и космоса [140], а также в фармацевтике, поскольку картирование различных комплексов в таблетке является важной проблемой для производственного контроля качества, а также для обнаружения подделок. Например, было показано [141], что возможно измерить распределение нескольких неорганических элементов $(\mathrm{Zn}, \mathrm{Fe}, \mathrm{Ti}, \mathrm{Mn}, \mathrm{Cu})$ до глубины в несколько сотен микрон под поверхностью таблетки, исключив „вмешательство“ в анализ покрытия таблетки, и в том же эксперименте измерить толщину покрытия.

Иногда необходимо знание толщины и состава внешнего слоя (оболочки) объекта исследования. Например, в работе [142] в зернах риса был обнаружен внешний слой, имеющий толщину около $80 \mu \mathrm{m}$, обогащенный металлами. Поэтому в регионах, на которые воздействуют тяжелые металлы и другие ядовитые элементы загрязнения, приготовление рисовых блюд нуждается в обильном количестве воды для обработки риса и кулинарии.

\section{3. Фокусирующая пористая рентгеновская оптика}

\section{1. Геометрия Вольтера I}

Идея использования ПВО для рентгеновской оптики родилась в 1960 г., когда Р. Джаккони и Б. Росси оценили возможность создания рентгеновского рефлектора усеченной параболической формы [143], способного сфокусировать параксиальный пучок РЛ без сферической аберрации.

Параболическая форма тем не менее не может быть использована для создания телескопов на основе ПВО, поскольку на них будет влиять сильная кома-аберрация; в результате поле зрения оптики будет мало для создания любого изображения в фокальной плоскости.

В 1952 г. Вольтер продемонстрировал двойную зеркальную систему, в которой используемые для формирования изображения РЛ претерпевали два последовательных отражения от параболоида, а затем гиперболоида [6]. Преимуществом двойной зеркальной системы является уменьшение оптических аберраций, таких как кома и, следовательно, улучшение углового разрешения. Но из-за удаленности большинства астрономических объектов интенсивность излучения на входе рентгеновского телескопа, как правило, чрезвычайно мала. Так как угол скольжения должен быть меньше критического угла ПВО, эффективная площадь одиночного зеркала очень мала. Для увеличения эффективной площади телескопа используются несколько коаксиальных и конфокальных зеркал с уменьшающимися радиусами („зеркальные оболочки“'), вложенные друг в друга наподобие русской матрешки, причем угол скольжения уменьшается от внешней оболочки к внутренней (рис. 8) [144,145].

Высокое угловое разрешение стоит дорого: зеркала должны иметь точно выдержанную форму поверхности с высоким качеством обработки и жесткое крепление. 


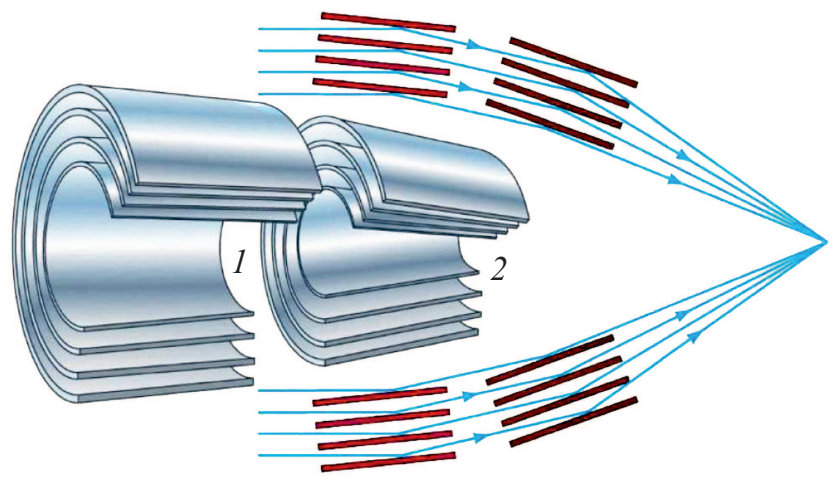

Рис. 8. Ход рентгеновских лучей в многослойном зеркале Вольтера І. При фокусировке лучи последовательно отражаются от параболической (1) и гиперболической (2) поверхностей каждой зеркальной оболочки.

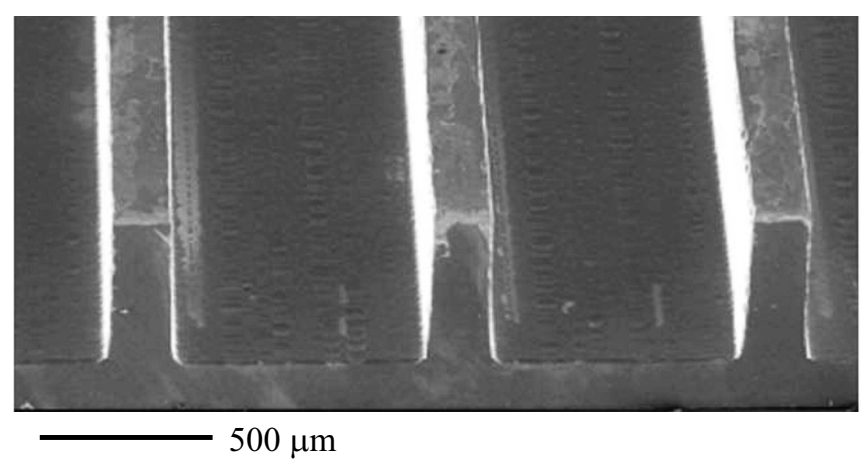

Рис. 9. Участок кремниевой пластины с ребрами жесткости шириной $0.17 \mathrm{~mm}$, шагом $1 \mathrm{~mm}$ и толщиной мембраны $0.17 \mathrm{~mm}$ [7].

Эти требования приводят к толстым оболочкам, высокой массе и большим затратам. С другой стороны, переход к более легким тонким зеркальным оболочкам делает оптику менее жесткой. Это уменьшение жесткости делает зеркало более восприимчивым к случайным нагрузкам и искажениям, которые могут ухудшить его форму. Следовательно, изготовление и монтаж тонких легких элементов рентгеновской фокусирующей оптики с высоким угловым разрешением представляет собой значительную и уникальную техническую проблему для современной астрофизики [146].

Для будущих рентгеновских телескопов необходимо уменьшить массу и объемную плотность упаковки зеркальных оболочек, сохраняя при этом эффективность, точную юстировку и жесткость конструкции.

Было показано [147], что стеклянный микропористый материал и миллипористые структуры кремния образуют ,жизнеспособные“ компоненты рентгеновской оптики. Обе технологии основаны на имеющихся в продаже продуктах и технологических процессах. Испытания демонстрационных линз показали, что их эффективность может быть приемлемой для легкой, компактной стек- лянной оптики и линзы более высокого разрешения и эффективности в случае кремниевой оптики.

\section{Кремниевая пористая оптика}

Было показано [7,144,147-150], что кремниевая пористая оптика (КПО) может служить новой технологией для создания следующего поколения рентгеновских телескопов. Выбор кремния в качестве материала для фокусирующей оптики не случаен: кремний имеет небольшую плотность $\left(2.3 \mathrm{~g} / \mathrm{cm}^{3}\right)$, обладает хорошей прочностью, малым температурным коэффициентом линейного расширения, и может быть отполирован с чрезвычайно высоким качеством. Процесс производства кремниевых пластин оптимизирован полупроводниковой промышленностью, что облегчает производство зеркал с заданными параметрами.

Полированные кремниевые пластины прямоугольной формы прорезаются канавками, чтобы сформировать ребристую структуру (рис. 9). Они имеют две основные функциональные части: мембрану, действующую как зеркало, и ребра для соединения нескольких пластин (рис. 10,a), расстояние между которыми может быть $\sim 1 \mathrm{~mm}$. Одна стенка каждой поры используется в качестве рабочей (отражающей) поверхности, а боковые стенки придают конструкции особую жесткость. Для улучшения отражательной способности зеркал их рабочая поверхность покрывается металлической пленкой. Форма поверхности зеркала, требуемая для оптики Вольтера I (или ее конического приближения), получается путем изгиба и укладки нескольких зеркал друг на друга, образуя зеркальный блок КПО (рис. 10,b). Важным моментом является то, что пластины соединены без какого-либо клея посредством гидрофильного связывания активированных поверхностей. Затем для того, чтобы получить конфигурацию Вольтера I, два блока КПО располагаются друг за другом, юстируются и соединяются в зеркальный модуль КПО (рис. 10,c). Апертура рентгеновского телескопа заполняется сотнями зеркальных модулей, объединенных в сектора [7] (рис. $10, d, e)$ или систему концентрических колец [144]. Таким образом, может быть достигнута жесткость конструкции за счет монолитной пористой структуры оптических модулей, которые индивидуально сьюстированы в оптический модуль, заменяя подход к установке отдельных оболочек, используемых в более традиционных рентгено-оптических технологиях. Это позволяет толщине подложки быть значительно уменьшенной, что приведет к более плотной упаковке зеркальных оболочек и тем самым к увеличению эффективной поверхности сбора без ущерба для жесткости оптики и точности формы.

\section{Стеклянная микропористая оптика}

Один из наиболее перспективных подходов для создания легкой и эффективной фокусирующей оптики - использование микропористых (микроканальных) пластин. Ширины отверстий в них настолько малы $(20-100 \mu \mathrm{m})$, что толщина подложки, т. е. длина зеркала, может быть 

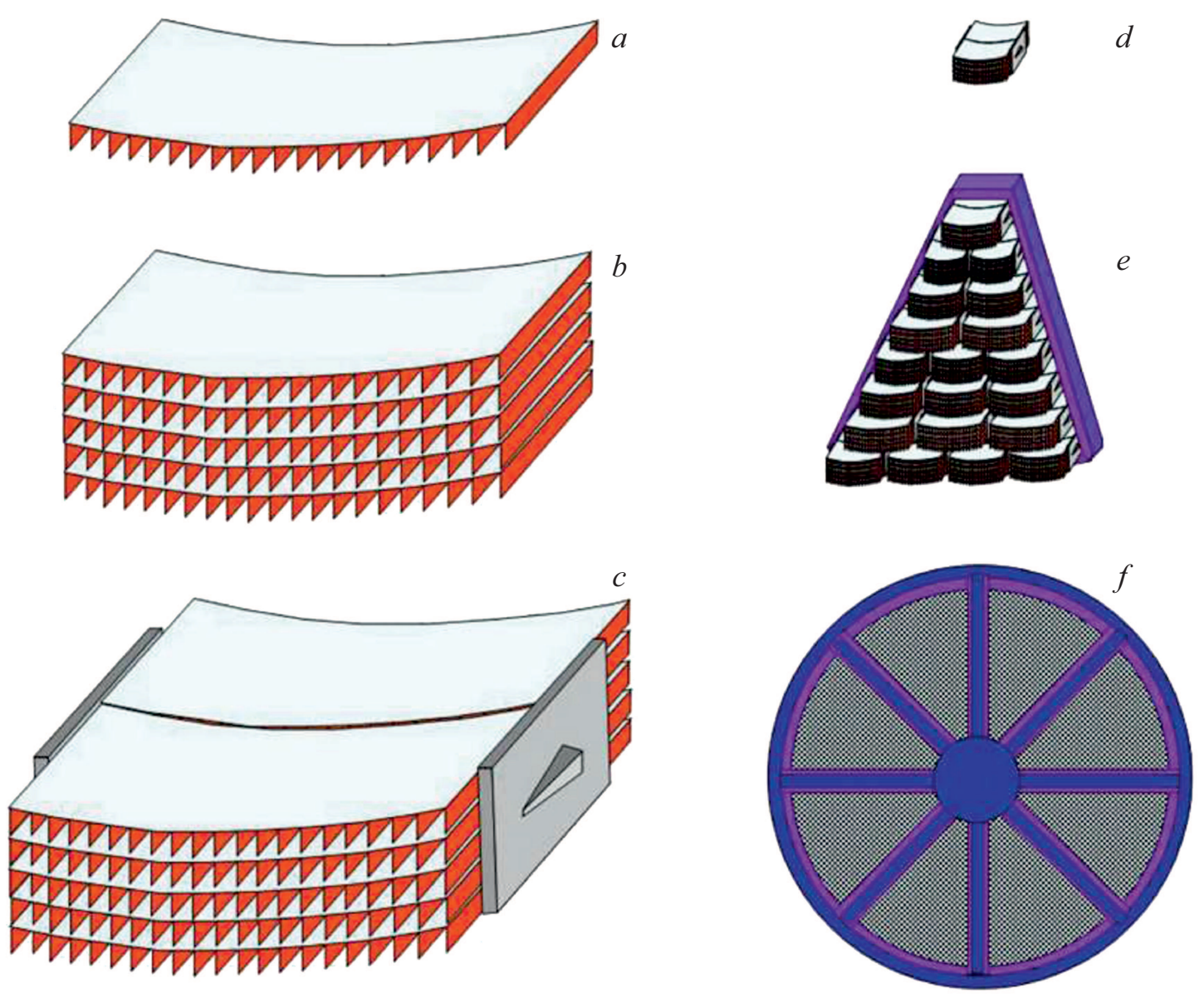

Рис. 10. Иллюстрация технологии изготовления КПО. Изогнутые зеркальные пластины с ребрами жесткости $(a)$ объединяются в зеркальные блоки $(b)$; два блока КПО соединяются скобами для формирования зеркального модуля оптики Вольтера I $(c, d)$; модули заполняют сектора (e), формирующие объектив телескопа $(f)$ [7].

небольшой, от сотен микрометров до десятков миллиметров. В результате оптика с микропорами может быть более чем в десять раз легче традиционной оптики рентгеновского телескопа. Таким образом, микропористая оптика при условии улучшения ее углового разрешения может быть использована для телескопов малой массы с большой эффективной площадью [151].

Микроканальные пластины (МКП) [7,148,152-157] изготавливаются из полированных стеклоблоков, окруженных облицовочным стеклом с более низкой температурой плавления. Блоки растягивают в тонкие волокна. Эти волокна объединяются в массив круглого или квадратного сечения, который снова вытягивается для формирования мультифибра. Для создания радиально упакованных МКП мультифибры расположены концентрическими кольцами и сплавлены при высоких температуре и давлении для образования жгута. Заготовки МКП толщиной несколько миллиметров нарезаются из жгута перед тем, как растворимое стекло сердцевины вытравливается кислотой, что приводит к желаемой структуре микропор требуемого сечения, в которой стенки пор образованы стеклом облицовки. МКП покрываются тонким слоем элемента с высоким $Z$ (например, платиной, иридием и т.д.) для повышения эффективности ПВО. В зависимости от приложения оптике может быть термически предана сферическая форма определенного радиуса. Поэтому технологию можно использовать для имитации двух поверхностей вращения, составляющих коническое приближение геометрии Вольтера I для рентгеновской визуализации $[154,155,158]$. Для этого требуется две пластины, съюстированные так, чтобы каналы в первой пластине совпадали с таковыми во второй и фиксировались вместе, чтобы сформировать структуру, показанную на рис. 11. Пластины должны быть изогнуты до сферической формы, где радиус кривизны второй пластины составляет 1/3 от радиуса первой пластины. РЛ от источника, расположенного на большом расстоянии, отражаются последовательно первой и второй пластиной, а изображение формируется на фокусном расстоянии $1 / 4$ радиуса кривизны первой пластины [7,154]. Такая оптика может обеспечить большую эффективную площадь при очень малой массе [154].

Реализация МКП предполагалась в программе полета миссии BepiColombo и миссии SVOM [159]. Для 


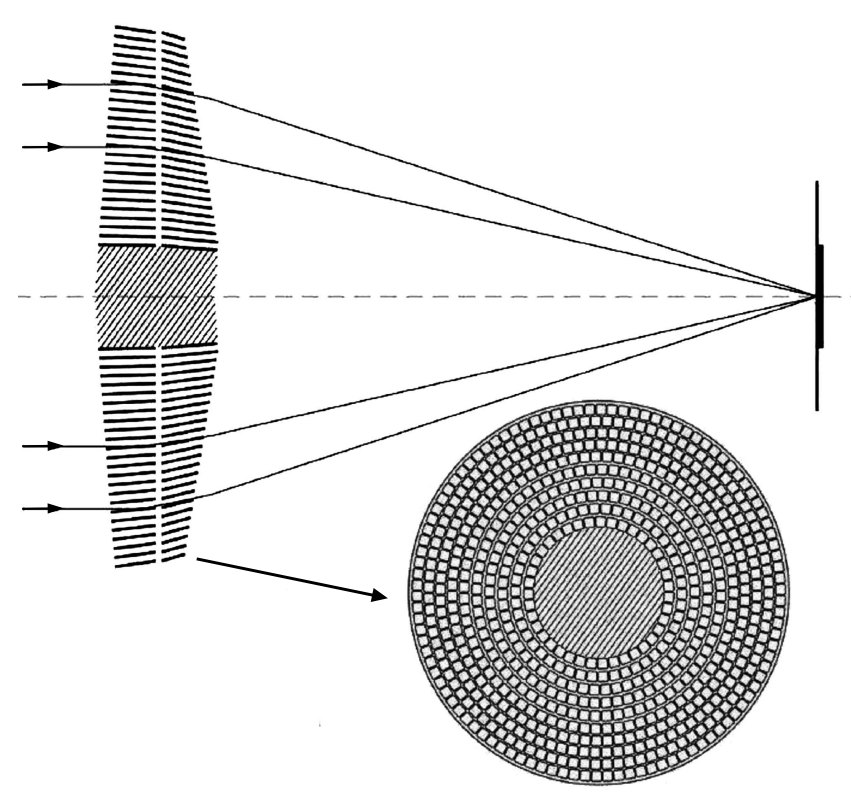

Рис. 11. Оптика Вольтера I может быть реализована с использованием большого количества прямоугольных пор, расположенных концентрическими кругами вокруг сердечника [148].

радиально упакованной оптики МКП миссии SVOM с квадратными порами размером $20 \mu \mathrm{m}$ в коническом приближении геометрии Вольтера I фокусное расстояние равно $1 \mathrm{~m}$, что определяется радиусами кривизны 4 и $1.33 \mathrm{~m}$ передней и задней пластин тандемной пары МКП. Оптика диаметром $210 \mathrm{~mm}$ собрана из тандемных пар МКП, каждая из которых представляет собой сектор окружности. Тандемы расположены в три кольца с разной толщиной (2.2 mm внутренняя, $1.3 \mathrm{~mm}$ средняя и $0.9 \mathrm{~mm}$ внешняя), чтобы приблизиться к профилю идеальной толщины, которая увеличивает пропускную способность телескопа за счет максимальной вероятности однократного отражения в каждой МКП [159].

Кремниевая микропористая оптика

Кремниевая микропористая оптика - это так называемые микро- электро- механические системы (МЭМС) с использованием анизотропного влажного травления пластин кремния с ориентацией поверхности (110) [160]. Поскольку они изготавливаются на основе литографии, доступны поры точной формы в масштабе $1 \mu \mathrm{m}$. Этот тип микропористой оптики может быть самой легкой рентгеновской оптикой благодаря мелким порам большой плотности. Так как травление позволяет изготавливать ряд рентгеновских зеркал сразу за один процесс травления, технология отличается очень низкой стоимостью.

Микропористая оптика МЭМС, изготовленная методом глубокого реактивного ионного травления, страдает высокой шероховатостью боковых стенок микроканалов $(10-30 \mathrm{~nm})$. Для улучшения характеристик трехмерных микроструктур МЭМС в работе [161] было предложено комбинированное использование процессов сухого и анизотропного влажного травления [160] кремниевой микроканальной рентгеновской оптики: первый - для придания микроструктуре требуемой оптической формы, второй - для получения гладких стенок каналов.

Для уменьшения шероховатости стенок каналов авторы работ $[162,163]$ применили метод водородного отжига при температуре $1000-1300^{\circ} \mathrm{C}$. Среднеквадратичная шероховатость поверхности после водородного отжига может быть чрезвычайно мала (порядка $0.1 \mathrm{~nm}$ ).

В работах $[164,165]$ описывается процесс сверхточной полировки до шероховатости $<3 \mathrm{~nm}$ с использованием переменного магнитного поля. Основным фактором, определяющим успех полировки, является воздействие абразивных частиц на заготовку силой магнитного поля. Было обнаружено, что процесс в основном контролируется частотой колебаний и напряженностью магнитного поля.

Для изготовления оптики Вольтера I, очевидно, следует изогнуть микроканальную пластину в зеркальный блок и соединить две пластины с разным радиусом кривизны. Однако в случае кремниевой структуры упругая деформация затруднена из-за хрупкости и жесткости кремния. В этом случае возможно использовать технологию горячей пластической деформации кремния. Монокристаллическую кремниевую пластину можно пластически деформировать в трехмерную форму путем помещения пластины между вогнутым и выпуклым штампами при высокой температуре $\left(>700^{\circ} \mathrm{C}\right)$ [166]. Рентгеновский телескоп, использующий тонкие кремниевые пластины с большим количеством мелких пор в качестве фокусирующей оптики, может стать самым легким из когда-либо созданных [163].

\section{2. Оптика „глаза лобстера“}

Мониторинг больших областей неба представляет особый интерес в рентгеновской астрономии из-за сильной изменчивости во времени большинства источников рентгеновского излучения. Ожидается, что широкопольные рентгеновские телескопы с фокусирующей оптикой станут важным инструментом в будущих проектах космической астрономии. Рентгеновская оптика, предложенная в 70-е годы Шмидтом [167] и Энджелом [168] дает прекрасную возможность получить очень широкое поле зрения (1000 квадратных градусов и более), в то время как обычно используемые классические зеркала скользящего падения Вольтера I ограничены примерно $1^{\circ}[8]$.

Двумерная система Шмидта состоит из ортогональных стопок плоских фольг, расположенных веером по периметру двух цилиндров с радиусами $R_{1}, R_{2}$ (рис. 12,a). Оптику Энджела можно рассматривать как особый случай системы Шмидта, где обе стопки лежат в том же положении, но имеют одинаковые радиусы $R_{1}=R_{2}=R$ (рис. 12,b). При этом две стопки зеркал образуют квадратные поры (рис. $12, c$ ). 
$a$

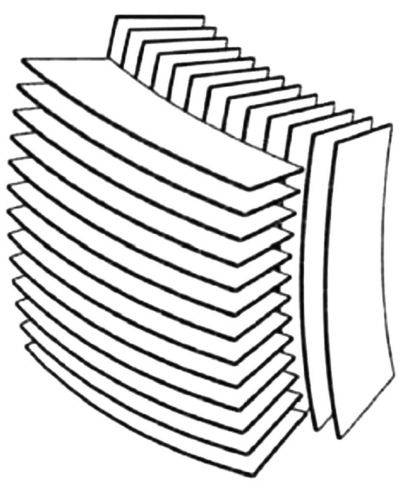

$b$

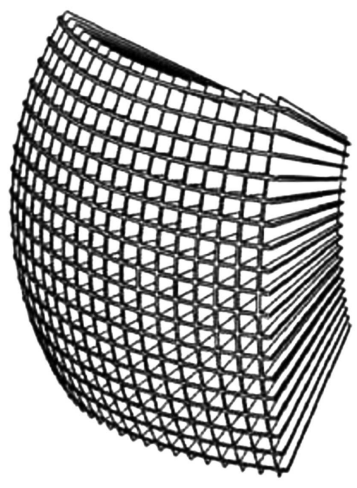

$c$

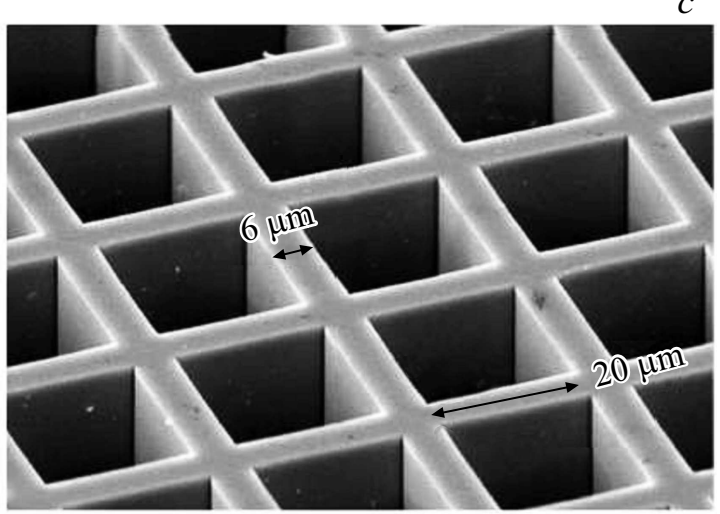

Рис. 12. Двумерная фокусирующая оптика в геометрии Шмидта $(a)$ и Энджела $(b)$. РЭМ-изображение микропор $(c)$.

Здесь нас будет интересовать оптика Энджела, которую принято называть оптикой „глаза лобстера“ (ГЛ) и которая образует отдельный класс пористой оптики. Дело в том, что глаза омаров и других ракообразных (креветок, раков и т.д.) устроены иначе, чем у большинства других животных: они не преломляют, а отражают падающий на них свет. То есть для фокусировки света на сетчатке глаза используются не линзы, каковыми являются хрусталики глаз высших позвоночных, а множество крошечных каналов с отражающими внутренними стенками и почти идеально квадратного сечения, расположенных на сферической поверхности. Такая система обладает чрезвычайно высокой светочувствительностью, практически недостижимой при использовании обычных линз.

Особый интерес к оптике ГЛ проявили астрофизики $[8,169-174]$.

Микроканальные пластины с квадратными порами и квадратной упаковкой (рис. 12,c) изготавливаются путем вытягивания, плавления и травления волокон свинцового стекла [175]. Оптика ГЛ представляет собой микроканальную пластинку, расположенную на сфере с радиусом $R$ (рис. $12, b, 13, a$ ). Однако в отличие от рассмотренной выше оптики МКП и МЭМС, в которой фокус образуют однократно отраженные в каждой пластинке рентгеновские лучи, в оптике ГЛ фокус формируется лучами, последовательно отраженными от соседних (ортогональных) стенок канала. Эти лучи концентрируются на фокальной поверхности радиусом $R / 2$. Линза ГЛ генерирует крестообразную форму фокуса с ветвями, возникающими в результате отражений фотонов от одной стенки канала. В результате структура фокуса представляет собой яркое пятно с более слабым крестом и гораздо менее интенсивным диффузным фоном, образованным РЛ, прошедшими линзу без отражений (рис. 13, $b$ ).

Недостаток оптики ГЛ - необходимость в регистрирующих изображение детекторах большого размера, устанавливаемых на фокальной поверхности.
Геометрическое угловое разрешение $\Delta \theta_{g}$ может оцениваться как отношение размера канала $d$ к фокусному расстоянию $f(f=R / 2)$ :

$$
\Delta \theta_{g}=2 d / R
$$

Наконец, поле зрения FoV объектива с $N \times N$ каналами определяется диапазоном углов, который формируется всеми каналами [176]:

$$
\mathrm{FoV} \approx N(2 d / R)=N \Delta \theta_{g} .
$$

Размер поля зрения зависит только от угловых размеров сферической оптики и детектора. Если оптика и детектор покрывают полусферу, поле обзора - все небо. Поэтому такая оптика идеально подходит для очень широкого применения в астрономии, несмотря на низкую разрешающую способность.

Если параллельный пучок падает на линзу ГЛ, апертура ограничена квадратом со стороной, равной $2 R \theta_{c}$. Поэтому самая большая достижимая эффективная область дается формулой [176]:

$$
\left(A_{\mathrm{eff}}\right)_{\max }=4\left(R \theta_{c}\right)^{2} .
$$

Чтобы получить максимально эффективное поле зрения, требуется $\theta_{c} \approx(180 / \pi)\left(8^{1 / 2}+1\right) d / L$ градусов $(L-$ осевая длина канала), поэтому при использовании материала покрытия высокой плотности, такого как иридий, при энергии РЛ $1 \mathrm{keV}$ оптимальное соотношение $L / d=50$. Производятся микрокапилярные пластинки со стандартными размерами пор обычно 20 или $40 \mu \mathrm{m}$, так что толщина пластин находится в диапазоне $1-2 \mathrm{~mm}$.

В работе [177] были выявлены все существенные аберрации, которые ограничивают характеристики микроканальных пластин с квадратными порами, используемых в качестве рентгеновской оптики ГЛ. Существуют три собственные аберрации, которые ограничивают угловое разрешение. Сферическая аберрация зависит от аспектного отношения $(L / d)$ и дает угловое разрешение $\Delta \theta_{s}=32^{1 / 2}(d / L)^{3}$, геометрический размер 

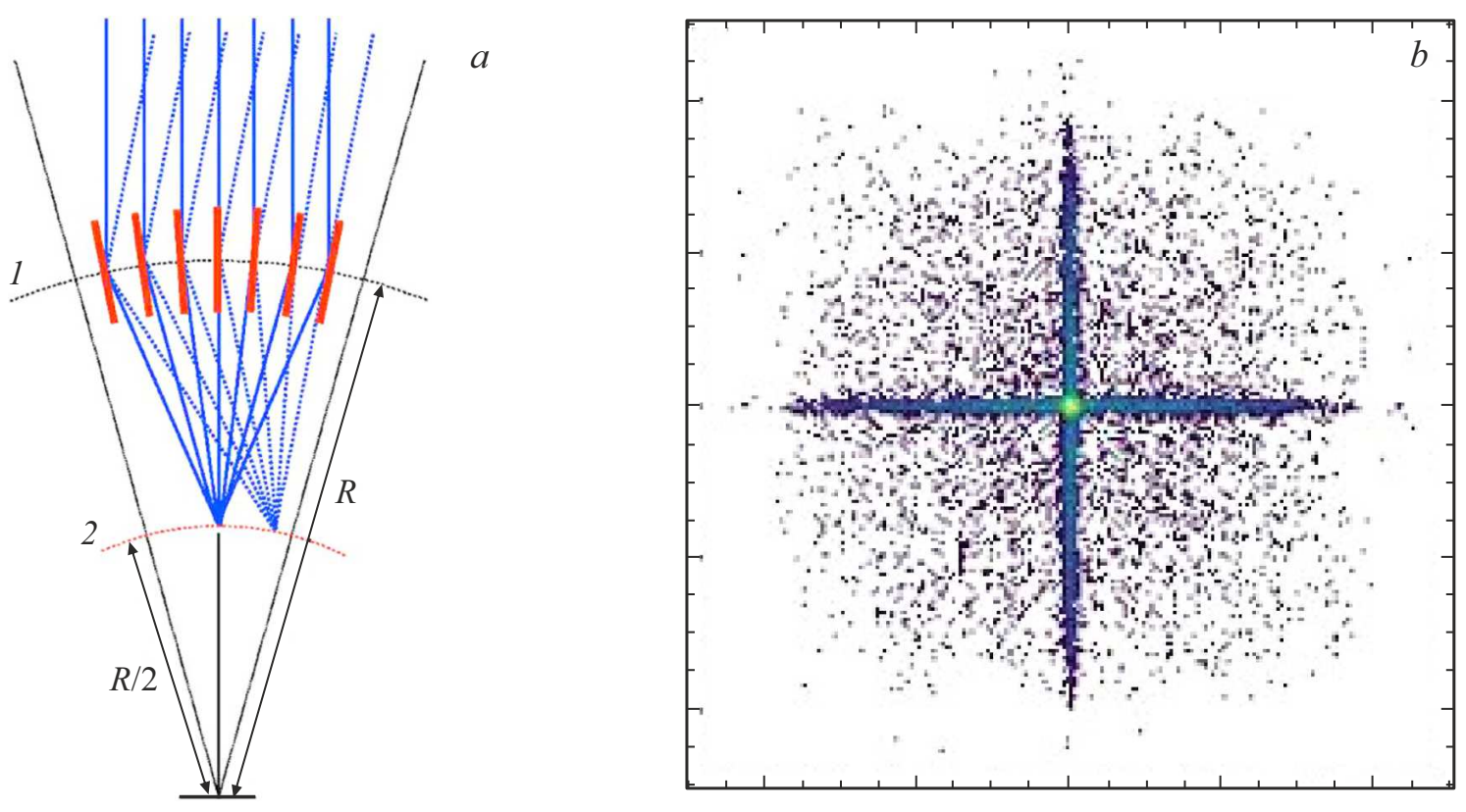

Рис. 13. Схема оптики ГЛ, показывающая рентгеновские зеркальные ячейки 1 , установленные на сферической поверхности с радиусом $R$ и фокальную поверхность 2 на фокусном расстоянии $R / 2$. Рентгеновское излучение от разных положений источников на небе фокусируется в разных местах фокальной поверхности $(a)$. Крестообразная форма фокуса оптики ГЛ $(b)$ [174].

пор ограничивает угловое разрешение до $\Delta \theta_{g}=d / f$ и дифракция ограничивает угловое разрешение до $\Delta \theta_{d}=2 \lambda / d$. Использование оптимального аспектного отношения $L / d=50$ дает предел сферической аберрации $\Delta \theta_{s} \approx 9^{\prime \prime}$. При отсутствии внешних аберраций будет получено максимальное угловое разрешение, если размер пор выбран таким, чтобы геометрические и дифракционные пределы были равны. В этом случае требуется $d=(2 \lambda f)^{1 / 2}$, что дает $\Delta \theta_{g}=\Delta_{d}=(2 \lambda / f)^{1 / 2}$. При $E=1 \mathrm{keV}, f=1 \mathrm{~m}$ и $d=50 \mu \mathrm{m} \Delta \theta_{g}=\Delta \theta_{d}=10^{\prime \prime}$. Если объединить сферические, геометрические и дифракционные пределы, получим собственное угловое разрешение $\left(\Delta \theta_{g}^{2}+\Delta \theta_{d}^{2}+\Delta \theta_{s}^{2}\right)^{1 / 2}=\Delta \theta_{i} \approx 17^{\prime \prime}$. Повторяя этот расчет для $f=0.3 \mathrm{~m}$ и используя ту же самую величину $L / d$, получим оптимальный размер пор $d=27 \mu \mathrm{m}$, $\Delta \theta_{g}=\Delta \theta_{d}=19^{\prime \prime}$ и общий предел углового разрешения $\Delta \theta_{i}=28^{\prime \prime}$

Размер пор $d$ доступных стеклянных микроканальных пластинок хорошо согласован с пределами оптимального углового разрешения геометрии ГЛ для рентгеновского телескопа в энергетическом диапазоне $0.1-10 \mathrm{keV}$. Они могут быть изготовлены с толщиной $L$ в диапазоне 0.9-4 mm, что дает оптимальное значение $L / d \sim 50$ для эффективной рентгенографии в том же диапазоне энергии. При формировании изображений в узком поле зрения предел собственного углового разрешения составит 10-20", а для более коротких фокусных расстояний, больше подходящих для приложений, требующих широкого поля зрения, предел будет находиться в диапазоне $20-30^{\prime \prime}$ [177].

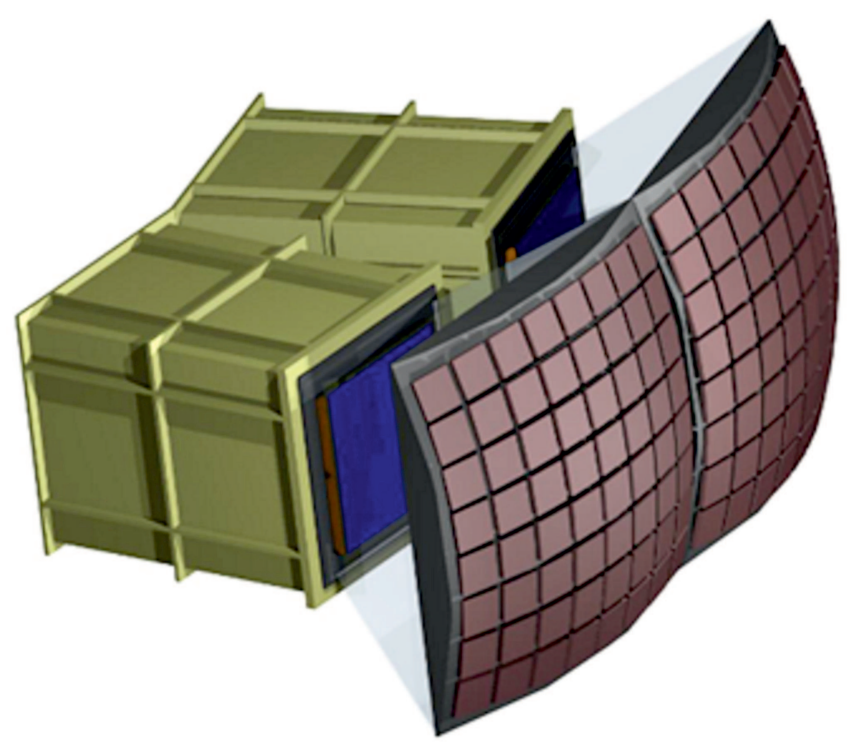

Рис. 14. Общий вид двух из четырех модулей оптики ГЛ сканера SXI миссии THESEUS [189] (см. текст).

Однако угловое разрешение также в значительной степени зависит от производственных ошибок и внешних факторов. Поэтому ряд работ был посвящен оптимизации оптических характеристик линзы ГЛ [173,174,178-182]. Например, авторами работы [178] были предложены способы оптимизации технологии подготовки и вытяжки стекла, контроля производственных процессов и метрологии, проведено усовершенство- 
вание процессов укладки волокон, травления сердцевины стекла и термического изгиба микроканальных пластин, а также методы покрытия стенок каналов отражающей пленкой. Для улучшения углового разрешения в работе [180] было рекомендовано применять терморегулирование оптики ГЛ. Для получения высокого аспектного отношения, радиально ориентированных прямоугольных каналов, уменьшения разориентации каналов и шероховатости их поверхностей (последние являются основными факторами предельного разрешения и отражательной способности пористой оптики) авторами работ $[181,182]$ предлагается использование рентгеновской литографии как возможной конкурирующей технологии изготовления микропористой оптики.

Оптическая система ГЛ была использована в нескольких научных миссиях, нацеленных на мониторинг всего неба: Lobster-ISS [183], AXIOM [184], STORM [185,186], SMILE [187], SVOM [188], THESEUS [189].

Например, апертура оптики рентгеновского сканера SXI миссии THESEUS образована массивом из $8 \times 8$ микроканальных пластинок с квадратными порами площадью $40 \times 40 \mathrm{~mm}^{2}$ каждая (рис. 14). Микроканальные пластинки установлены на сферической рамке с радиусом кривизны $600 \mathrm{~mm}$. Фокальная плоскость каждого модуля сканера представляет собой сферическую поверхность радиусом кривизны $600 \mathrm{~mm}$, расположенную на расстоянии $300 \mathrm{~mm}$ (фокусное расстояние) от апертуры оптики. Детекторы для каждого модуля состоят из массива $2 \times 2$ широкоформатных детекторов, наклоненных таким образом, чтобы приблизиться к сферической фокальной поверхности.

\section{Заключение. Выводы и перспективы}

Следует констатировать, что наиболее распространенным и удобным способом фокусировки первичного рентгеновского излучения на сегодняшний день является использование капиллярной и поликапиллярной рентгеновской оптики. За счет умеренного разрешения поликапиллярные элементы обеспечивают высокую интенсивность и часто используются как концентраторы рентгеновских лучей с синхротронами и рентгеновскими трубками. В сочетании с микрофокусными рентгеновскими трубками капиллярные линзы позволяют создавать компактные приборы для неразрушающего локального элементного анализа.

Одним из возможных путей дальнейшего развития поликапиллярной оптики может стать придание ей функции изображающей оптики. Недавно была предложена дефектная микроскопия [190], использующая естественные дефекты поликапиллярных структур, такие как разрушенные, отсутствующие или чуть более крупные капилляры. Было продемонстрировано, что такие дефекты, нарушая периодичность капиллярных решеток, приводят непосредственно к формированию множественных рентгеновских изображений объекта, помещенного в фокусе поликапиллярной оптики. Множественные изображения могут быть проанализированы с использованием принципа кодирующей апертуры и обещают обеспечить пространственное разрешение на уровне $0.5 \mu \mathrm{m}$. Метод очень перспективен, но для практических приложений требует сложного изготовления специальной рентгеновской оптики с намеренно внесенными дефектами. Поэтому в работе [191] была предложена субмикронная кодирующая апертурная микроскопия с использованием внешней периодической сетки, расположенной на выходной поверхности поликапиллярной оптики.

Впечатляют достижения в области создания легкой рентгеновской оптики в геометрии Вольтера I на основе микропористых пластин. Однако здесь предстоит большая работа, направленная на существенное улучшение углового разрешения. Кроме этого, существует проблема юстировки двух микропористых пластин разной кривизны с большим количеством каналов. Поэтому может оказаться перспективной предложенная авторами работ [192,193] новая компактная и легкая стеклянная микропористая рентгеновская оптика, которая с помощью фемтосекундного лазерного излучения и влажного травления формирует систему Вольтера I в единой стеклянной подложке без ее изгиба и необходимости юстировки.

Широкоугольная рентгеновская оптика „глаза лобстера“ вышла на уровень, позволяющий ее успешное использование в орбитальных телескопах. Недавние новые разработки показали, что оптика „глаза лобстера“ также подходит для рентгеновской визуализации неастрономических объектов [194] и может использоваться в медицине, системе безопасности и для борьбы с терроризмом. Например, портативный аппарат LEXID („Lobster-Eye“ X-ray Inspection Device), разработанный компанией Physical Optics Corporation (POS) по заказу министерства внутренней безопасности США, основан на регистрации рентгеновского излучения, рассеянного изучаемым объектом, и может видеть сквозь бетонные стены и стальные заграждения толщиной в несколько сантиметров с расстояния до $2.5 \mathrm{~m}$ [195]. Устройство в первую очередь предназначено для таможенников.

Другая разработка этой же фирмы - прибор LEXIUS - представляет собой систему подводного рентгеновского сканера/рефлектометра для получения высококачественных изображений мин и других объектов, скрытых донными отложениями.

Вывод: фокусирующая рентгеновская капиллярная и пористая оптики являются широко используемыми высокоэффективными техниками, обладающими способностями к дальнейшему развитию и совершенствованию.

\section{Финансирование работы}

Работа выполнена при поддержке Министерства науки и высшего образования в рамках выполнения работ по Государственному заданию ФНИЦ „Кристаллография и фотоника“ РАН. 


\section{Список литературы}

[1] Ice G.E., Budai J.D., Pang J.W.L. // Science. 2011. V. 334. N 6060. P. 1234.

[2] Schmahl G., Rudolph D., Schneider G., Guttmann P., Niemann B. // Optik. 1994. V. 97. N 4. P. 181.

[3] Guttmann P., Zeng X., Feser M., Heim S., Yun W., Schnei$\operatorname{der}$ G. // J. Phys.: Conf. Ser. 2009. V. 186. N 1. P. 012064.

[4] Howells M., Jacobsen C., Warwick T., Bos A. // Principles And Applications of Zone Plate X-Ray Microscopes. Science of Microscopy. NY.: Springer, 2007. V. 2. P. 835.

[5] Wolter H. // Ann. Phys. 1952. B. 10. S. 94.

[6] Wolter H. // Ann. Phys. 1952. B. 10. S. 286.

[7] Bavdaz M., Collon M., Beijersbergen M., Wallace K., Wille E. // X-ray Opt. Instrum. 2010. V. 2010. ID 295095.

[8] Priedhorsky W.C., Peele A.G., Nugene K.A. //Mon. Not. Roy. Astron. Soc. 1996. V. 279. N 3. P. 733.

[9] Дабагов С.Б. // УФН. 2003. Т. 173. N 10. С. 1083.

[10] Furuta K., Nakayama Y., Shoji M., Kaigawa R., Hanamoto K., Nakano H., Hosokawa Y. // Rev. Sei. Instrum. 1993. V. 64. N 1. P. 135.

[11] Wang L., Rath B.K., Gibson W.M., Kimball J.C., MacDonald C.A. // J. Appl. Phys. 1996. V. 80. N 7. P. 3628.

[12] Chen G.J., Cerrina F., Voss K.F., Kim K.H., Brown F.C. // Nucl. Instrum. Methods Phys. Res. A. 1994. V. 347. N 1-3. P. 407.

[13] Ullich J.B., Kovantsev V.E., MacDonald C.A. // J. Appl. Phys. 1993. V. 14. N 10. P. 5933.

[14] Аркадьев В.А., Коломийцев А.И., Кумахов М.А., Пономарев И.Ю., Ходеев И.А., Чертов Ю.П., Шахпаронов И.М. // УФН. 1989. Т. 157. № 3. С. 529.

[15] MacDonald C.A., Gibson W.M. // X Ray Spectrom. 2003. V. 32. P. 258.

[16] Suparmi Cari, Wang L., Wang H., Gibson W.M., MacDonald C.A. // J. Appl. Phys. 2001. V. 90. N 10. P. 5363.

[17] Сторижко В.Е., Ильяшенко М.В., Молодкин В.Б., Гаевский А.Ю., Денисенко В.Л., Денисенко О.И., Вершинский C.A. // Успехи физ. мет. 2010. Т. 11. С. 1.

[18] Bilderback D.H. // X-Ray Spectrom. 2003. V. 32. P. 195.

[19] Charnley N.R., Potts P.J., Long J.V.P. // J. Anal. At. Spectrom. 1994. V. 9. N 11. P. 1185.

[20] Zeng X., Duewer F., Feser M., Huang C., Lyon A., Tkachuk A., Yun W. // Appl.Opt. 2008. V. 47. N 13. P. 2376.

[21] Bjeoumikhov A., Bjeoumikhova S., Wedell R. // Part. Part. Syst. Charact. 2005. V. 22. N 6. P. 384.

[22] De Nolf W., Janssens K. // Surf. Interface Anal. 2010. V. 42. N 5. P. 411.

[23] Snigireva I., Snigirev A. // J. Environ. Monit. 2006. V. 8. N 1. P. 33.

[24] Thiel D.J., Bilderback D.H., Lewis A., Stern E.A. // Nucl. Instrum. Methods Phys. Res. A. 1992. V. 317. N 3. P. 597.

[25] Bilderback D.H., Hoffman S.A., Thiel D.J. // Science. 1994. V. 263. N 5144. P. 201.

[26] Hoffman S.A., Thiel D.J., Bilderback D.H. // Nucl. Instrum. Methods Phys. Res. A. 1994. V. 347. N 1-3. P. 384.

[27] Vincze L., Janssens K., Adams F., Rindby A., Engstrijm P., Riekel C. // Adv. X-Ray Anal. 1999. V. 41. P. 252.

[28] Balaic D.X., Barnea Z., Nugent K.A., Garrett R., Varghese R.F., Wilkins S.W. // J. Synchrotron Rad. 1996. V. 3. P. 289.

[29] Chen J., Wu C., Tian J., Li W., Yu S., Tian Y. // Appl. Phys. Lett. 2008. V. 92. N 23. P. 233104.
[30] Sorrentino A., Nicolás J., Valcárcel R., Chichón F.J. // J. Synchrotron Rad. 2015. V. 22. N 4. P. 1112.

[31] Huang R., Szebenyi T., Pfeifer M., Woll A., Smilgies D.M., Finkelstein K., Dale D., Wang Y., Vila-Comamala J., Gillilan R., Cook M., Bilderback D.H. // J. Phys.: Conf. Ser. 2014. V. 493. P. 012034

[32] Engström P., Larsson S., Rindby A., Stocklassa B. // Nucl. Instrum. Methods Phys. Res. B. 1989. V. 36. N 2. P. 222.

[33] Merkle A., Gelb J., Lavery L. // Microsc. Microanal. 2013. V. 19. N S2. P. 1314.

[34] Müller M., Mey T., Niemeyer J., Mann K. // Opt. Express. 2014. V. 22. N 9. P. 23489.

[35] Benk M., Bergmann K., Schäfer D., Wilhein T. // Opt. Lett. 2008. V. 33. N 20. P. 2359.

[36] Vincze L., Somogyi A., Osan J., Vekemans B., Török S., Janssens K., Adams F. // Anal. Chem. 2002. V. 74. N 5. P. 1128.

[37] Bartoll J., Röhrs S., Erko A., Firsov A., Bjeoumikhov A., Langhoff N. // Spectrochim. Acta B. 2004. V. 59. N 10-11. P. 1587.

[38] Limburg K.E., Lochet A., Driscoll D., Dale D.S., Huang R. // X-ray Spectrom. 2007. V. 36. N 5. P. 336.

[39] Lopes R.T., Lima I., Pereira G.R., Perez C.A. // Pramana. 2011. V. 76. N 2. P. 271.

[40] Mroczka R., Żukociński G., Lopucki R. //Proc. SPIE. 2017. V. 10235. P. 10235OE.

[41] Lind Q.C., De Nolf W., Janssen K., Salbu B. // J. Environ. Radioact. 2013. V. 123. P. 63.

[42] Wang J., Chen-Wiegart Y., Wang J. // Nat. Commun. 2014. V. 5. Art. 4570.

[43] Merkle A., Gelb J. // Microscopy Today. 2013. V. 21. N 2. P. 10.

[44] Tan C., Daemi S.R., Heenan T. M.M., Iacoviello F., Leach A., Rasha L., Jervis R., Brett D., Shearing P. // J. Electrochem. Soc. 2020. V. 167. N 6. P. 060512.

[45] Li R., Cornaby S., Kamperman M., Smilgiers D.M. // J. Synchrotron Rad. 2011. V. 18. N 5. P. 697.

[46] Sirenko A.A., Kazimirov A., Huang R., Bilderback D.H., O'Malley S., Gupta V., Bacher K., Ketelsen L.J.P., Ougazzaden A. // J. Appl. Phys. 2005. V. 97. N 6. P. 063512.

[47] Li F., Liu Z., Sun T. // J. Appl. Cryst. 2016. V. 49. N 2. P. 627.

[48] Kazimirov A., Bilderback, Huang R., Sirenko A., Ougazzaden A. // J. Phys. D: Appl. Phys. 2004. V. 37. N 4. P. L1.

[49] Pantell R.H., Chung P.S. // IEEE J. Quant. Electron. 1978. V. 14. N 9. P. 694.

[50] Бушуев В.А., Оруджалиев М.Н., Кузьмин Р.Н. // ЖТФ. 1989. V. 59. N 11. P. 153.

[51] Kumakhov M.A. // Nucl. Instrum. Methods Phys. Res. B. 1990. V. 48. N 1-4. P. 283.

[52] Kumakhov M.A., Komarov F.F. // Phys. Rep. 1990. V. 191. N 5. P. 289.

[53] Kumakhov M.A. // X-ray Spectrom. 2000. V. 29. N 5. P. 343.

[54] Gao N., Janssens K. Polycapillary X-ray optics/ in: X-Ray Spectrometry: Recent Technological Advances, Wiley, Chichester, 2004. P. 89.

[55] MacDonald C.A. // X-Ray Opt. Instrum. 2010. V. 2010. Art. 867049.

[56] Болотоков А., Зайцев Д., Лютиау А., Щербаков А. // Аналитика. 2012. № 4. С. 14. 
[57] Beloglazov V., Langhoff N., Tuchin V., Bjeoumikhov A., Bjeoumikhova Z., Wedel R., Skibina N., Skibina Y.S., Chainikov M. // J. X-Ray. Sci. Technol. 2005. V. 13. N 4. P. 179.

[58] MacDonald C.A., Petruccelli J.C. // J. Phys.: Conf. Ser. 2016. V. 776. P. 012001

[59] Wegrzynek D., Mroczka R., Markowicz A., Chinea-Cano E., Bamford S. // X-Ray Spectrom. 2008. V. 37. N 6. P. 635.

[60] Sun T., Liu Z., Li Y., Wang G., Zhu G., Ding X., Xu Q., Liu H., Luo P., Pan Q., Lin X., Teng Y. // Spectrochim. Acta B. 2009. V. 64. N 11-12. P. 1194.

[61] MacDonald C.A., Gibson W.M. // Proc. SPIE. 1995. V. 2519. P. 186.

[62] Sun T., MacDonald C.A. // J. Appl. Phys. 2013. V. 113. P. 053104.

[63] Sun T., MacDonald C.A. // J. X-Ray Sci. Technol. 2015. V. 23. N 2. P. 141.

[64] Bashir S., Tahir S., MacDonald C., Petruccelli J.C. // Opt. Commun. 2016. V. 369. P. 28.

[65] Owens S.M., Ullrich J.B., Ponomarev I., Carter D.C., Sisk R.C., Ho J.X., Gibson W.M. // Proc. SPIE. 1996. V. 2859. P. 200.

[66] MacDonald C.A., Owens S.M., Gibson W.M. // J. Appl. Cryst. 1999. V. 32. N 26. P. 160.

[67] Gao N., Ponomarev I., Xiao Q.F., Gibson W.M., Carpenter D.C. // Appl. Phys. Lett. 1996. V. 69. N 11. P. 1529.

[68] Gao N., Ponomarev I., Xiao Q.F., Gibson W.M., Carpenter D.C. // Appl. Phys. Lett. 1997. V. 71. N 23. P. 3441.

[69] Yan Y., Gibson W.M. // Adv. X-Ray Anal. 2002. V. 45. P. 298.

[70] Alfeld M., Pedroso J.V., van E. Hommes M., Van der Snickt G., Tauber G., Blaas J., Haschke M., Erler K., Dikb J., Janssens K. // J. Anal. At. Spectrom. 2013. V. 8. N 5. P. 760.

[71] Bronk H., Rhrs S., Bjeoumikhov A., Langhoff N., Schmalz J., Wedell R., Gorny H.-E., Herold A., Waldschläger U. // Fresenius J. Anal. Chem. 2001. V. 371. N 3. P. 307.

[72] Haschke M., Rossek U., Tagle R., Waldschlager U. // Adv. X-Ray Anal. 2012. V. 55. P. 286.

[73] Trentelman K., Bouchard M., Ganio M., Namowicz C., Patterson C.S., Walton M. // X-Ray Spectrom. 2010. V. 39. N 3. P. 159.

[74] Trentelman K., Janssens K., van der Snickt G., Szafran Y., Woollett A.T., Dik J. // Appl. Phys. A. 2015. V. 121. P. 801.

[75] Martins A., Albertson C., McGlinchey C., Dik J. // Herit. Sci. 2016. V. 4. P. 22.

[76] Martins A., Coddington J., Geert van der Snickt, van Driel B., McGlinchey C., Dahlberg D., Janssens K., Dik J. // Herit. Sci. 2016. V. 4. P. 33.

[77] Duivenvoorden J.R., Käyhkö A., Kwakkel E., Dik J. // Herit. Sci. 2017. V. 5. P. 6.

[78] Hrnjića M., A. Hagen-Petera G., Bircha T., Barfod G.H., Sindbak S.M., Lesher C.E. // Nucl. Instrum. Methods Phys. Res. B. 2020. V. 478. P. 11.

[79] Vincze L., Wei F., Proost K., Vekemans B., Janssens K., He Y., Yan Y., Falkenberg G. // J. Anal. At. Spectrom. 1997. V. 17. N 3. P. 177.

[80] Hofmann A., Freinberg-Truffas C.A., Osens S.M., Padiyar S.D., MacDonald C.A., // Nucl. Instrum. Methods Phys. Res. B. 1997. V. 133. N 1-4. P. 145.

[81] Proost K., Vincze L., Janssens K., Gao N., Bulska E., Schreiner M., Falkenberg G. // X Ray Spectrom. 2003. V. 32. N 3. P. 215.
[82] Tomik B., Chwiej J., Szczerbowska-Boruchowska M., Lankosz M., Wójcik S., Adamek D., Falkenberg G., Bohic S., Simionovici A., Stegowski Z., Szczudlik A. // Neurochem. Res. 2006. V. 31. N 3. P. 321.

[83] Sun T., Liu Z., He B., We S.i, Xie Y., Liu T., Hu T., Ding X. // Nucl. Instrum. Methods Phys. Res. A. 2007. V. 574. N 2. P. 285.

[84] Sun T., Zhang M., Liu Z., Zhang Z., Li G., Ma Y., Du X., Jia $Q$., Chen Y., Yuan Q., Huang $W$., Zhu P., Ding X. // J. Synchrotron Rad. 2009. V. 16. N 1. P. 116.

[85] Surowka A.D., Wrobel P., Adamek D., Radwanska E., Szczerbowska-Boruchowska M. // Metallomics. 2015. V. 7. N 11. P. 1522.

[86] Gibson W.M., Kumakhov M.A. // Proc. SPIE. 1993. V. 1736. P. 172.

[87] Николаев В.И., Чижсова Е.В. // Научное приборостроение. 2011. Т. 21. № 2. С. 3.

[88] Ding X., Gao N., Havrilla G. // Proc. SPIE. 2000. V. 4144. P. 174.

[89] Nakano K., Tsuji K. // J. Anal. At. Spectrom. 2010. V. 25. N 4. P. 562.

[90] Sun T., Ding X. // Rev. Anal. Chem. 2015. V. 34. P. 45.

[91] Woll A.R., Agyeman-Budu D., Choudhury S., Coulthard I., Finnefrock A.C., Gordon R., Hallin E., Mass J. // J. Phys.: Conf. Ser. 2014. V. 493. P. 12028.

[92] Chen G., Chu S., Sun T., Sun X., Zheng L., An P., Zhu J., $W u$ S., Du Y., Zhang J. // J. Synchrotron Rad. 2017. V. 24. N 5. P. 1000.

[93] Hampai D., Cherepennikov Yu.M., Liedl A., Cappuccio G., Capitolo E., Iannarelli M., Azzutti C., Gladkikh Yu.P., Marcellia A., Dabagov S.B. // JINST. 2018. V. 13. N 4. P. C04024.

[94] Bauters S., Tack P., Rudloff-Grund J.H., Banerjee D., Longo A., Vekemans B., Bras W., Brenker F.E., Van Silfhout R., Vincze L. // Anal. Chem. 2018. V. 90. N 3. P. 2389.

[95] Tsuji K., Nakano K. // X-Ray Spectrom. 2007. V. 36. N 3. P. 145.

[96] Nakazawa T., Tsuji K. // X-Ray Spectrom. 2013. V. 42. N 5. P. 374.

[97] Kanngiesser B., Malzer W., Rodrigues A.F., Reiche I. // Spectrochim. Acta B. 2005. V. 60. N 1. P. 41.

[98] Xiaoyan L., Zhihong W., Tianxi S., Qiuli P., Xunliang D. // Nucl. Instrum. Methods Phys. Res. B. 2008. V. 266. N 11. P. 2638

[99] Mantouvalou I., Lange K., Wolff T., Grötzsch D., Lühl L., Haschke M., Hahn O., Kanngiesser B. // J. Anal. At. Spectrom. 2010. V. 25. N 4. P. 554.

[100] Laclavetine K., Ager F.J., Arquillo J., Respaldiza M.Á., Scrivano S. // Microchem. J. 2016. V. 125. P. 62.

[101] it Xiang Z.J., Meng Z.Y., Liu Z.G., Pan K., Zhao W.G., Zhou P., Li Y. // IOP Conf. Ser.: Mater. Sci. Eng. 2020. V. 770. P. 012054.

[102] Ingerle D., Swies J., Iro M., Wobrauschek P., Streli C., Hradil K. // Rev. Sci. Instrum. 2020. V. 91. N 12. P. 123107.

[103] Kanngiesser B., Malzer W., Mantouvalou I., Sokaras D., Karydas A.G. // Appl. Phys. A. 2012. V. 106. N 2. P. 325.

[104] Tsuji K., Nakano K. // J. Anal. At. Spectrom. 2011. V. 26. N 2. P. 305.

[105] Senkbeil T., Mohamed T., Simon R., Batchelor D., Di Fino A., Aldred N., Clare A.S., Rosenhahn A. // Anal. Bioanal. Chem. 2016. V. 408. N 5. P. 1487. 
[106] Seim C., Laurenze-Landsberg C., Schröder-Smeibidl B., Mantouvalou I., de Boerd C., Kanngiesser B. // J. Anal. At. Spectrom. 2014. V. 29. N 8. P. 1354.

[107] Šmit Ž., Janssens K., Proost K., Langus I. // Nucl. Instr. Methods Phys. Res B. 2004. V. 219-220. N 1. P. 35.

[108] Kanngiesser B., Malzer W., Reiche I. // Nucl. Instrum. Methods Phys. Res. B. 2003. V. 211. N 2. P. 259.

[109] Woll A.R., Bilderback D. H., Gruner S., Gao N., Huang R., Bisulca C., Mass J. // Mater. Res. Soc. Symp. Proc. 2005. V. 852. P. 281.

[110] Woll A. R., Mass J., Bisulca C., Cushman M., Griggs C., Wazny T., Ocon N. // Studies in Conservation. 2008. V. 53. N 2. P. 93.

[111] Malzer $W$. // The Rigaku J. 2006. V. 23. P. 40.

[112] Reiche I., Müller K., Eveno M., Itié E., Menu M. // J. Anal. At. Spectrom. 2012. V. 27. N 10. P. 1715.

[113] Reiche I., M?ller K., Mysak E., Eveno M., Mottin B. // Appl. Phys. A. 2015. V. 121. N 3. P. 903.

[114] Nakano K., Tabe A., Shimoyama S., Tsuji K. // Microchem. J. 2016. V. 126. P. 496.

[115] Reiche I., Eveno M., Mülle K.r, Calligaro T., Pichon L., Laval E., Mysak E., Mottin B. // Appl. Phys. A. 2016. V. 122. N 11. P. 947.

[116] Zhou P., Liu Z., Ma X., Meng Z., Xiang Z., Wang X., Sun T., Lin X., Li Y. // Nucl. Instrum. Methods Phys. Res. B. 2020. V. 464. P. 111.

[117] Kanngiesser B., Mantouvalou I., Malzer W., Wolff T., Hahn O. // J. Anal. At. Spectrom. 2008. V. 23. N 6. P. 814.

[118] Nakano K., Tsuji K. // X-Ray Spectrom. 2009. V. 38. N 5. P. 446.

[119] Yi L., Qin M., Wang K., Lin X., Peng S., Sun T., Liu Z. // Appl. Phys. A. 2016. V. 122. N 9. P. 856.

[120] Nakazawa T., Tsuji K. // X-Ray Spectrom. 2013. V. 42. N 3. P. 123.

[121] Qin M., Y L.i, Wang J., Han Y., Sun T., Liu Z. // IOP Conf. Ser.: Mater. Sci. Eng. 2017. V. 269. P. 012033.

[122] Nakano K., Akioka K., Doi T., Arai M., Takabe H., Tsuji K. // ISIJ International. 2013. V. 53. N 11. P. 1953.

[123] Akioka K., Nakazawa T., Doi T., Arai M., Tsuji K. // Powder Diffr. 2014. V. 29. N 2. P. 151.

[124] Menzel M., Schlifke A., Falk M., Janek J., Fröba M., Fittschen U.E.A. // Spectroc. Acta B. 2013. V. 85. P. 62.

[125] Baker A.M., Cai Y., Ziegelbauer J.M., Agyeman-Budu D., Woll A., Kongkanand A., Mukundan R., Borup R.L. // ECS Trans. 2019. V. 92. N 8. P. 107.

[126] Tsuji K., Yonehara T., Nakano K. // Anal. Sci. 2008. V. 24. N 1. P. 99.

[127] Perez R. D., Sanchez H. J., Perez C. A., Rubio M. // Radiat. Phys. Chem. 2010. V. 79. N 2. P. 195.

[128] McCormick N., Velasquez V., Finney L., Vogt S., Kelleher S.L. // PLoS ONE. 2010. V. 5. P. e11078.

[129] Rauwolf M., Turyanskaya A., Roschger A., Prost J., Simon R., Scharf O., Radtke M., Schoonjans T., Guilherme Buzanich A., Klaushofer K., Wobrauschek P., Hofstaetter J. G., Roschgerb P., Streli C. // J. Synchrotron Rad. 2017. V. 24. N 1. P. 307.

[130] Vincze L., Vekemans B., Brenker F.E., Falkenberg G., Rickers K., Somogyi A., Kersten M., Adams F. // Anal. Chem. 2004. V. 76. N 22. P. 6786.

[131] Schmitz S., M?ller A., Wilke M., Malzer W., Kanngiesser B., Bousquet R., Berger A., Schefer S. // Eur. J. Mineral. 2009. V. 21. N 5. P. 927.
[132] Jiang B., Zhu Y., Sun T., Liu Z., Li F., Sun X., Wang Y., Ding K. // Spectrosc. Lett. 2017. V. 50. N 10. P. 545.

[133] Choudhury S., Swanston T., Varney T.L., Cooper D.M.L., George G.N., Pickering I.J., Grimes V., Bewer B., Coulthard I. // Archaeometry. 2016. V. 58. N S1. P. 207.

[134] Choudhury S., Agyeman-Budu D.N., Woll A.R., Swanston T., Varney T.L., Cooper D.M.L., Hallin E., George G.N., Pickering I.J., Coulthard I. // J. Anal. At. Spectrom. 2017. V. 32. N 3. P. 527.

[135] Hampai D., Liedl A., Cappuccio G., Capitolo E., Iannarelli M., Massussi M., Tucci S., Sardella R., Sciancalepore A., Polese C., Dabagov S.B. // Nucl. Instrum. Methods Phys. Res. B. 2017. V. 402. P. 274.

[136] Nakano K., Nishi C., Otsuki K., Nishiwaki Y., Tsuji K. //Anal. Chem. 2011. V. 83. N 9. P. 3477.

[137] Emoto S., Otsuki K., Nakano K., Tsuji K. // Adv. X-Ray Anal. 2013. V. 56. P. 217.

[138] Sun X., Zhang X., Wang X., Wang Y., Li Y., Peng S., Shao S., Liu Z., Shang H., Sun T. // X-Ray Spectrom. 2020. V. 49. N 2. P. 267.

[139] Fittschen U.E.A., Falkenberg G. //Anal. Bioanal. Chem. 2011. V. 400. N 6. P. 1743.

[140] Schmitz S., Brenker F.E., Schoonjans T., Vekemans B., Silversmit G., Vincze L., Burghammer M., Riekel C. // Geochim. Cosmochim. Acta 2009. V. 73. N 6. P. 5483.

[141] Mazel V., Reiche I., Busignies V., Walter P., Tchoreloff P. // Talanta. 2011. V. 85. N 1. P. 556.

[142] Mihucz V.G., Silversmit G., Szalóki I., de Samber B., Schoonjans T., Tatár E., Vincze L., Virág I., Yao J., Záray G. // Food Chem. 2010. V. 121. N 1. P. 290.

[143] Giacconi R., Rossi B. // J. Geophys. Res. 1960. V. 65. P. 773.

[144] Wille E., Bavdaz M. // Acta Astronautica. 2015. V. 116. N 1. P. 50.

[145] Saha T.T., McClelland R.S., Zhang W.W. // Proc. SPIE. 2014. V. 9144. P. 914418.

[146] DeRoo C.T., Allured R., Cotroneo V., Hertz E., Marquez V., Reid P.B., Schwartz E.D., Vikhlinin A.A., Trolier-McKinstry S., Walker J., Jackson T.N., Liu T.N., Tendulkar M. // J. Astron. Telesc. Instrum. Syst. 2018. V. 4. N 1. P. 019004.

[147] Wallace K., Bavdaz M., Collon M., Beijersbergen M., Kraft S., Fairbend R., S?guy J., Blanquer P., Graue R., Kampf D. // Proc. SPIE. 2006. V. 10567. P. 105670U.

[148] Beijersbergen M., Kraft S., Bavdaz M., Lumb D., Günther R., Collon M., Mieremet A., Fairbend R., Peacock A. // Proc. SPIE. 2004. V. 5539. P. 104.

[149] Wille E., Wallace K., Bavdaz M., Collon M.J., Günther R., Ackermann M., Beijersbergen M.W., Riekerink M.O., Blom M., Lansdorp B., de Vreede L. // Proc. SPIE. 2017. V. 10565. P. 105652L.

[150] Westergaard N.J., Ferreira D.D.M., Massahi S. // Nucl. Instrum. Methods Phys. Res. A. 2017. V. 873. N 21. P. 5.

[151] Bavdaz M., Lumb D., Peacock A., Beijersbergen M., Kraft S. // Proc. SPIE. 2004. V. 5488. P. 829.

[152] Chapman H.N., Nugent K.A., Wilkins S.W., Davis T.J. // J. X-Ray Sci. Technol. 1990. V. 2. N 2. P. 117.

[153] Laprade B., Cochran R.C., Langevin F., Dykstra M.W. // Proc SPIE. 1997. V. 3173. P. 474.

[154] Willingale R., Fraser G.W., Brunton A.N., Martin A.P. // Exp. Astron. 1998. V. 8. N 4. P. 281. 
[155] Beijersbergen M.W., Bavdaz M., Peacock A.J., Tomaselli E., Fraser G., Brunton A., Flyckt E., Krumrey M.K., Souvorov A. // Proc. SPIE. 1999. V. 3765. P. 452.

[156] Collon M., Beijersbergen M., Wallace K., Bavdaz M., Fairbend R., Séguy J., Schyns E., Krumrey M., Freyberg M. // Proc. SPIE. 2007. V. 6688. P. 668812.

[157] Wallace K., Collon M., Beijersbergen M., Oemrawsingh S., Bavdaz M., Schyns E. // Proc. SPIE. 2007. V. 6688. P. 66881C.

[158] Price G.J., Brunton A.N., Beijersbergen M.W., Fraser G.W., Bavdaz M., Boutot J.P., Fairbend R., Flyckt S.O., Peacock A., Tomaselli E. // Nucl. Instrum. Methods Phys. Res. A. 2002. V. 490. N 1-2. P. 276.

[159] Gotz D., Osborne J., Cordier B., Paul J., Evans P., Beardmore A., Martindale A., Willingale R., O'Brien P., Basa S., Rossin C., Godet O., Webb N., Greiner J., Nandra K., Meidinger N., Perinati E., Santangelo A., Mercier K., Gonzalez F. // Proc. SPIE. 2014. V. 9144 . P. 914423.

[160] Ezoe Y., Koshiishi M., Mita M., Mitsuda K., Hoshino A., Ishisaki Y., Yang Z., Takano T., Maeda R. // Appl. Opt. 2006. V. 45. N 35. P. 8932.

[161] Ezoe Y., Koshiishi M., Mita M., Mitsuda K., Hoshino A., Ishisaki Y., Takano T., Maeda R. // Nucl. Instrum. Methods Phys. Res. A. 2007. V. 579. N 2. P. 817

[162] Ezoe Y., Mitsuishi I., Takagi U., Koshiishi M., Mitsuda K., Yamasaki N.Y., Ohashi T., Kato F., Sugiyama S., Riveros R.E., Yamaguchi H., Fujihira S., Kanamori Y., Morishita K., Nakajima K., Maeda R. // Microsyst. Technol. 2010. V. 16. N 8-9. P. 1633.

[163] Ezoe Y., Miyoshi Y., Kasahara S., Kimura T., Ishikawa K., Fujimoto M., Mitsuda K., Sahara H., Isobe N., Nakajima H., Ohashi T., Nagata H., Funase R., Ueno M., BranduardiRaymont G. // J. Astron. Telesc. Instrum. Syst. 2018. V. 4. N 4. P. 046001.

[164] Mitsuishi I., Ezoe Y., Takagi U., Mita M., Riveros R., Yamaguchi H., Kato F., Sugiyama S., Fujiwara K., Morishita K., Nakajima K., Fujihira S., Kanamori Y., Yamasaki N., Mitsuda K., Maeda R. // Proc. SPIE. 2009. V. 7360. P. 736040.

[165] Riveros R. E., Yamaguchi H., Mitsuishi I., Takagi U., Ezoe Y., Kato F., Sugiyama S., Yamasaki N., Mitsuda K. // Appl. Optics. 2010. V. 49. N 18. P. 3511.

[166] Nakajima K., Fujiwara K., Pan W., Okuda H. // Nat. Mater. 2005. V. 4. N 1. P. 47.

[167] Schmidt W.H.K. // Nucl. Instr. Meth. 1975. V. 127. P. 285.

[168] Angel J.R.P. // Astrophys. J. 1979. V. 233. P. 364.

[169] Chapman H.N., Nugent K.A., Wilkins S.W. // Rev. Sci. Instr. 1991. V. 62. N 6. P. 1542.

[170] Kaaret P., Geissbühler P. // Proc. SPIE. 1991. V. 1546. P. 82.

[171] it Kaaret P., Geissbuhler P., Chen A., Glavinas E. // Appl. Opt. 1992. V. 31. N 34. P. 7339.

[172] Peele A.G., Nugent K.A., Rode A.V., Gabel K., Richardson M.C., Strack R., Siegmund W. // Appl. Opt. 1996. V. 35. N 22. P. 4420.

[173] Su L., Li W., Wu M., Su Y., Guo C., Ruan N., Yang B., Yan F. // Appl. Opt. 2017. V. 56. N 22. P. 6267.

[174] Tamagawa T., Uchiyama K., Otsubo R., Yuasa T., Zhou Y., Mihara T., Ezoe Y., Numazawa M., Ishi D., Fukushima A., Suzuki H., Uchino T., Sakuta S., Ishikawa K., Enoto T., Sakamoto T. // J. Astron. Telesc. Instrum. Syst. 2020. V. 6. N 2. P. 025003.

[175] Wiza J.L. // Nucl. Instr. Meth. 1979. V. 162. P. 587.
[176] Grubsky V., Gertsenshteyn M., Shoemaker K., Jannson T. // Proc. SPIE. 2007. V. 6688. P. 66880P.

[177] Willingale R., Pearson J.F., Martindale A., Feldman C.H., Fairbend R., Schyns E., Petit S., Osborne J. P., O’Brien P. T. // Proc. SPIE. 2016. V. 9905. P. 99051Y.

[178] Mutz J.-L., Bonnet O., Fairbend R., Schyns E., Séguy J.// Proc SPIE. 2007. V. 6479. P. 64790F.

[179] Peng S., Wei F., Guo Y., Ye Y. // Opt. Eng. 2019. V. 58. N 9. P. 093101.

[180] Svendsen S., Knudsen E.B., Blake S., Oosterbroek T., Jegers A.S., Ferreira D.D.M., Prod'hommec T., Short B.t, Willingale R., O'Brien P. // Proc. SPIE. 2019. V. 11119. P. 111191R.

[181] Peele A.G., Irving T.H.K., Nugent K.A., Mancini D.C., Christenson T.R., Petre R., Brumby S.P., Priedhorsky W.C. // Rev. Sci. Instrum. 2001. V. 72. N 3. P. 1843.

[182] Peele A.G., Vora K.D., Shew B.-Y., Loechl B., Harvey E.C., Hayes J.P. // Microsyst. Technol. 2007. V. 13. N 5. P. 511.

[183] Fraser G.W., Brunton A.N., Bannister N.P., Pearson J.F., Ward M., Stevenson T.J., Watson D.J., Warwick B., Whitehead S., O'Brian P., White N., Jahoda K., Black K., Hunter S.D., Deines-Jones P., Priedhorsky W.C., Brumby S.P., Borozdin K.N., Vestrand T., Fabian A.C., Nugent K.A., Peele A.G., Irving T.H.K., Price S., Eckersley S., Renouf I., Smith M., Parmar A.N., McHardy I.M., Uttley P., Lawrence A. // Proc. SPIE. 2002. V. 4497. P. 115.

[184] Branduardi-Raymont G., Sembay S.F., Eastwood J.P., Sibeck D.G., Abbey A., Brown P., Carter J.A., Carr C.M., Forsyth C., Kataria D., Kemble S., Milan S.E., Owen C.J., Peacocke L., Read A.M., Coates A.J., Collier M.R., Cowley S.W.H., Fazakerley A.N., Fraser G.W., Jones G.H., Lallement R., Lester M., Porter F.S., Yeoman T.K. // Exp. Astron. 2012. V. 33. N 2. P. 403.

[185] Collier M.R., Scott Porter F., Sibeck D.G., Carter J.A., Chiao M.P., Chornay D.J., Cravens T.E., Galeazzi M., Keller J.W., Koutroumpa D., Kujawski J., Kuntz K., Read A.M., Robertson I.P., Sembay S., Snowden S.L., Thomas N., Uprety Y., Walsh B.M. // Rev. Sci. Instrum. 2015. V. 86. N 7. P. 071301.

[186] Walsh B.M., Collier M.R., Kuntz K.D., Porter F.S., Sibeck D.G., Snowden S.L., Carter J.A., Collado-Vega Y., Connor H.K., Cravens T.E., Read A.M., Sembay S., Thomas N.E. // J. Geophys. Res. Space Physics. 2016. V. 121. N 4. P. 3353.

[187] Raab W., Branduardi-Raymont G., Wang C., Dai L., Donovan E., Enno G., Escoubet P., Holland A., Jing L., Kataria D., Li L., Read A., Rebuffat D., Romstedt J., Runciman C., Sembay S., Spanswick E., Sykes J., Thornhill J., Wielders A., Zhang A., Zheng J. // Proc. SPIE. 2016. V. 9905. P. 990502.

[188] Feldman C., Pearson J., Willingale R., Sykes J., Drumm P., Houghton P., Bicknell C., Osborne J., Martindale A., O'Brien P., Fairbend R., Schyns E., Petit S., Roudot R., Mercier K., Le Duigou J., Gotz D. // Proc. SPIE. 2017. V. 10399. P. $103991 \mathrm{Q}$.

[189] Amati L., O’Brien P., Götz D., Bozzo E., Tenzer C., Frontera F., Ghirlanda G., Labanti C., Osborne J.P., Stratta G., Tanvir N., Willingale R., Attina P., Campana R., CastroTirado A.J., Contini C., Fuschino F., Gomboc A., Hudec R., Orleanski P. // Adv. Space Res. 2018. V. 62. N 4. P. 191. 
[190] Korecki P., Sowa K.M., Jany B.R., Krok F. // Phys. Rev. Lett. 2016. V. 116. N 23. P. 233902.

[191] Sowa K.M., Jany B.R., Korecki P. // Optica. 2018. V. 5. N 5. P. 577.

[192] Nomoto K., Hata R., Doi K., Nawaki Y., Yajima D., Furuya R., Shinoda K., Tsuruoka K., Kodaka H. // Proc. SPIE. 2018. V. 10760. P. 107600A.

[193] Doi K., Nomoto K., Nawaki Y., Uetsuki K., Hata R., Tsuruoka K., Kodaka H., Ito H., Harada Y., Asakawa Y. // Proc. SPIE. 2019. V. 11108. P. 11080V.

[194] Gertsenshteyn M., Jannson T., Savant G. // Proc. SPIE. 2005. V. 5922. P. 59220N.

[195] Ashcraft C. // Creation. 2010. V. 32. N 3. P. 21. 